\title{
Republic of Congo: Fifth and Sixth Reviews Under the Three-Year Arrangement Under the Extended Credit Facility and Financing Assurances Review-Staff Report; Staff Statement and Supplement; Press Release on the Executive Board Discussion; and Statement by the Executive Director for the Republic of Congo.
}

In the context of the fifth and sixth reviews under the Three-Year Arrangement under the Extended Credit Facility and Financing Assurances Review, the following documents have been released and are included in this package:

- $\quad$ The staff report for the Fifth and Sixth Reviews Under the Three-Year Arrangement Under the Extended Credit Facility and Financing Assurances Review, prepared by a staff team of the IMF, following discussions that ended on June 6, 2011, with the officials of the Republic of Congo on economic developments and policies. Based on information available at the time of these discussions, the staff report was completed on July 15, 2011. The views expressed in the staff report are those of the staff team and do not necessarily reflect the views of the Executive Board of the IMF.

- $\quad$ A staff supplement.

- $\quad$ A staff statement of July 27, 2011 and staff supplements of July 15, 2011 updating information on recent economic developments.

- $\quad$ A Press Release summarizing the views of the Executive Board as expressed during its July 27,2011 discussion of the staff report that completed the review.

- A statement by the Executive Director for the Republic of Congo.

The documents listed below have been or will be separately released.

Letter of Intent sent to the IMF by the authorities of the Republic of Congo* Technical Memorandum of Understanding*

*Also included in Staff Report

The policy of publication of staff reports and other documents allows for the deletion of market-sensitive information.

Copies of this report are available to the public from

International Monetary Fund • Publication Services $70019^{\text {th }}$ Street, N.W. • Washington, D.C. 20431

Telephone: (202) 623-7430 • Telefax: (202) 623-7201

E-mail: publications@imf.org Internet: http://www.imf.org

\section{International Monetary Fund Washington, D.C.}


INTERNATIONAL MONETARY FUND

\author{
REPUBLIC OF CONGO
}

\title{
Fifth and Sixth Reviews Under the Three-Year Arrangement Under the Extended Credit Facility and Financing Assurances Review
}

\author{
Prepared by the African Department \\ (in consultation with other departments)
}

Approved by Domenico Fanizza and Dhaneshwar Ghura

July 15, 2011

> Discussions were held in Brazzaville on March 22-30 and May 31-June 6, 2011. The mission met with President Sassou N'Guesso, State Minister Moussa (Planning), Finance Minister Ondongo, National Director of the BEAC Ondaye, other senior government officials, and representatives of the diplomatic and donor community, the private sector, and the media. The mission comprised Ms. Baker (head), Mr. Mpatswe, Ms. Tartari and Mr. Omar (all AFR), Mr. Acosta Ormaechea (FAD), and Ms. Rice and Mr. Ahouanmenou (all BLS) and was assisted by Mr. Melhado, resident representative. It was joined in March by Mr. Ondo Bile (OED).

$>\quad$ The ECF arrangement in the amount of SDR 8.46 million (10 percent of quota) was approved by the Executive Board on December 8, 2008. The arrangement is designed to achieve balanced growth, low and stable inflation and fiscal and external stability. The fourth review was completed in January 2011. The fifth review was delayed pending clarification of financing flows, including foreign deposits.

$>\quad$ Program implementation through end-December 2010 and end-March 2011 has been satisfactory, and the authorities observed all continuous, end-December and end-March quantitative performance criteria. The authorities also observed the two structural benchmarks related to oil governance, but fell short of the 80-percent target on procurement of public contracts through competitive bidding at both test dates due to early implementation difficulties at line ministries and the need to regularize previously signed contracts by bringing them under the new code. However, by end-May the authorities had achieved a rate of about 90 percent.

$>$ In the attached supplemental Letter of Intent (LOI), the authorities request completion of the fifth and sixth reviews under the ECF arrangement and have agreed to the publication of the LOI and this staff report. Upon completion of the sixth and final review, the program will expire.

$>$ Congo is a member of the Central African Economic and Monetary Community (CEMAC) and its regional central bank (BEAC). The common currency, the CFA franc, is pegged to the euro at CFAF 656. CEMAC member countries accepted the obligations of Article VIII in 1996. Congo maintains an exchange system free of restrictions on the making of payments and transfers for current international transactions. 


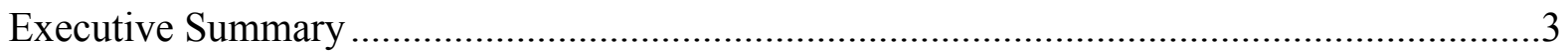

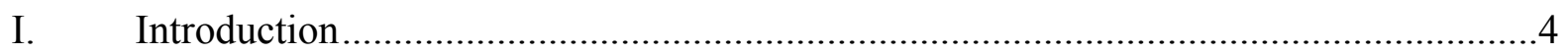

II. Developments, Program Performance and Outlook ....................................................

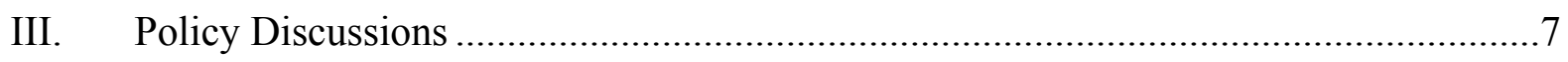

A. Fiscal policy-Sustained consolidation while making the most of investment .....7

B. Reforming the tax system and strengthening fiscal institutions .............................

C. Stepping up efforts to improve the business climate ...............................................11

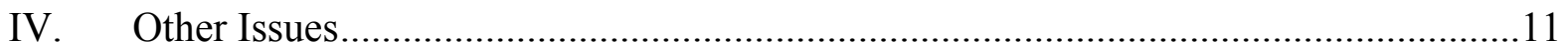

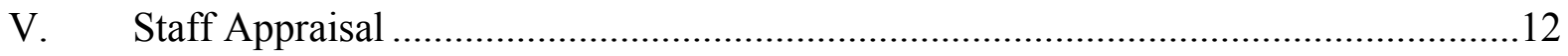

Box

1. Fiscal reform-Recommendations and actions planned for $2011 \ldots \ldots \ldots \ldots \ldots \ldots \ldots \ldots \ldots \ldots . . . . .10$

Figures

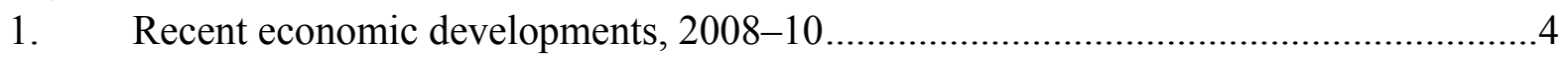

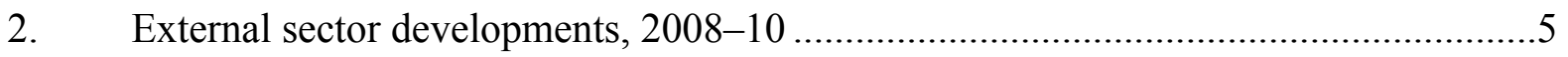

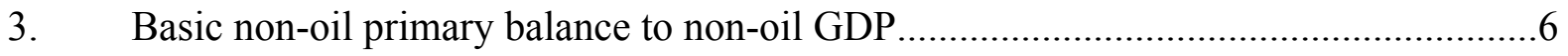

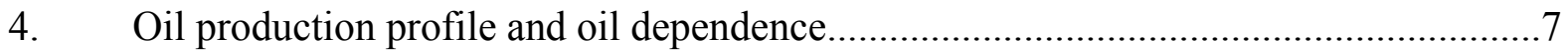

5. Non-oil tax revenue to GDP in select countries, 2006-10 ..........................................

\section{Text Table}

1. Quantitative Targets, 2010-11

Tables

1. Selected Economic and Financial Indicators, 2008-13 ……....................................14

2. Medium Term Balance of Payments, 2008-13 ......................................................15

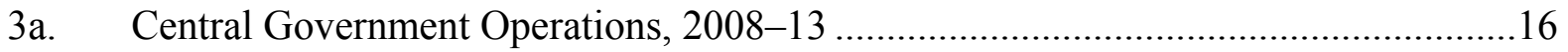

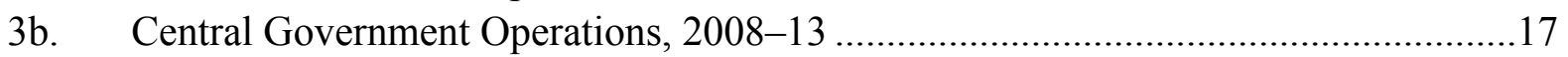

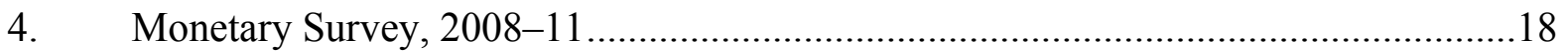

5. Quantitative Targets and projections, 2010-11 ...................................................19

6. Structural Benchmarks Under the ECF Arrangement, 2010-11 ...............................20

7. Access and Phasing Under the 3-year ECF Arrangement (2008-11).........................21

Appendix

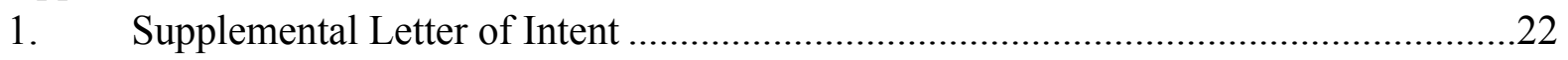

Attachment I. Technical Memorandum of Understanding ........................................2 


\section{EXECUTIVE SUMMARY}

\section{Recent Economic Developments and Prospects}

- Macroeconomic performance strengthened in 2010, mostly due to domestic factors, and has remained strong. There are signs that non-oil activity (construction, telecoms and forestry) continues to rise, while inflation has ticked up in line with global developments.

- The external position improved markedly in 2010. Bouncing oil prices shifted the current account into surplus, while debt relief following the HIPC Completion Point (January 2010) reduced external liabilities sharply. Gross official foreign assets rose to 44 percent of GDP at end-2010 and net debt is small at $3 \frac{1}{2}$ percent of GDP.

\section{Program Performance at end-December 2010 and end-March 2011}

- The authorities observed all continuous, end-December 2010 and end-March 2011 quantitative performance criteria. The cumulative adjustment of the basic non-oil primary deficit (BNOPD) over the first two years of the program was equivalent to the envisioned adjustment over the course of the full three-year ECF supported arrangement. The lower accumulation of government deposits in the regional central bank (BEAC) through March 2011 is explained mostly by higher foreign deposits, which are now being repatriated.

- The authorities observed the two structural benchmarks aimed at improving oil governance, while in the area of public financial management, the benchmark was met with delay. Despite significant progress in applying the new procurement code, they fell short of the targeted percentage (competitive bidding on 80 percent of contracts above $\$ 500,000$ ) on both test dates because of early implementation difficulties at the ministerial level and the need to regularize previously signed contracts by placing them under the new code. However, by end-May the authorities had achieved a rate of about 90 percent.

\section{Policies in the Period Ahead}

- Fiscal policy in 2011 focuses on scaling up investment spending on infrastructure, while continuing to save a sizable share of oil revenue. Measures are also in train to increase investment efficiency by strengthening public financial management. An advisor is in place to assist with project identification and assessment for the 2012 budget, and work is underway to further strengthen the expenditure chain.

- A fiscal reform program for 2011-13 aims to improve the design of Congo's tax system and strengthen fiscal institutions, while raising revenue collection. The authorities have already taken initial measures to unify tax rates and simplify the tax regime; are producing a tax expenditure document; and are working to strengthen capacity and reduce tax evasion. They are also gearing up to implement the action plan to improve the business climate. However, the uphill battle for more sweeping reforms continues, mostly due to vested interests.

Staff recommends completion of the fifth and sixth reviews under the ECF arrangement and disbursement of the sixth and seventh loans, in an amount equivalent to SDR 1,208,570 and SDR 1,208,580, respectively. 


\section{INTRODUCTION}

1. In 2011the authorities plan to scale up public investment to address longstanding development needs, while continuing to save a significant share of oil revenue. With oil production in mature fields expected to decline following its peak next year, policies aim to raise non-oil growth and reduce poverty through building a foundation of basic infrastructure and developing a business-friendly environment.

2. The authorities are confident that the policy framework laid out in their Memorandum of Economic and Financial Policies (MEFP, December 20, 2010) remains relevant, achievable and consistent with their policy objective of balancing development needs and fiscal sustainability. They are implementing action plans to strengthen public investment quality and the business climate, and taking measures to boost non-oil revenue while restraining current spending.

\section{Developments, Program Performance and Outlook}

3. Macroeconomic performance strengthened in 2010, largely as a result of domestic factors. Both oil production and non-oil activity (construction, telecoms, forestry) rose (Figure 1). Inflation ticked up in line with global developments, mitigated by positive supply shocks to agriculture and fixed domestic fuel prices. There are signs that activity in the nonoil sector continued to accelerate in the first quarter of 2011, while oil production declined by about 7 percent because of unscheduled stoppages.

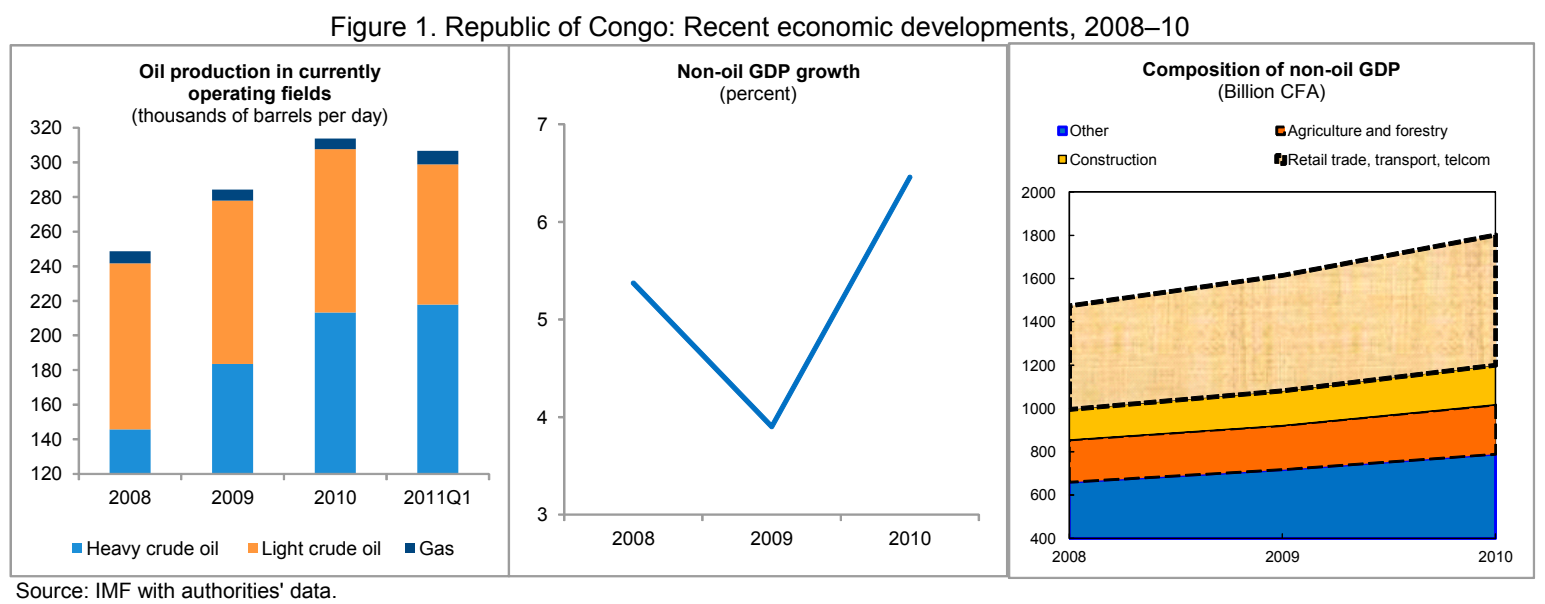

4. The external position further strengthened in 2010. The bounce in oil prices shifted the current account into surplus, while debt relief following the HIPC Completion Point (January 2010) reduced external liabilities significantly. Gross official foreign assets climbed. At end-2010 net debt dropped to 31/2 percent of GDP (Figure 2). 
Figure 2. Republic of Congo: External sector developments, 2008-10

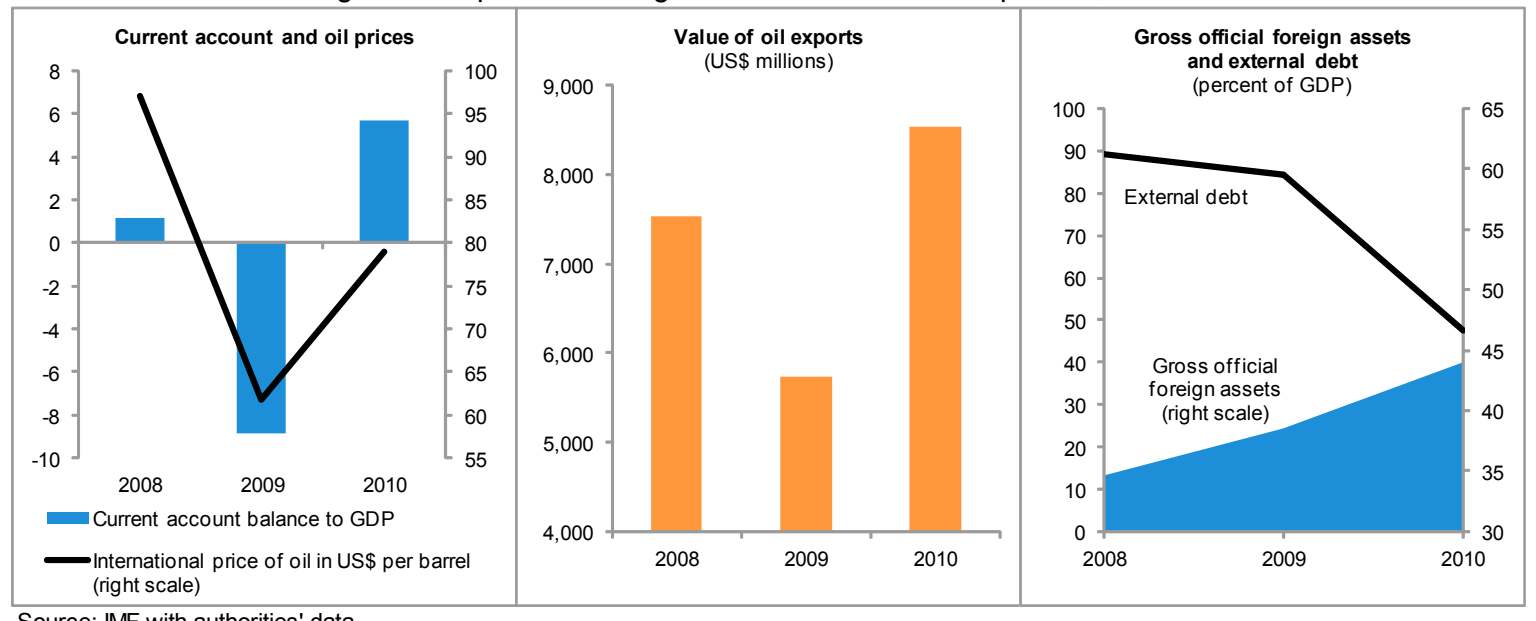

Source: IMF with authorities' data.

\section{The authorities observed all continuous, end-December 2010 and end-March 2011 performance criteria (Text Table 1).}

- The target on the basic non-oil primary deficit (BNOPD) was met. The higher (but still negative) net domestic financing of government relative to the indicative target is explained mostly by lower-than-envisioned oil revenues and higher accumulation of deposits abroad, which are now being repatriated. The fifth review was delayed pending clarification of financing flows, including foreign deposits.

\begin{tabular}{|c|c|c|c|c|c|c|}
\hline \multicolumn{7}{|c|}{$\begin{array}{c}\text { Text Table 1. Quantitative targets, 2010-11 } \\
\text { (Billions of CFA francs; cumulative from January; unless otherwise indicated) }\end{array}$} \\
\hline & \multicolumn{2}{|c|}{ End-Jun. 2010} & \multicolumn{2}{|c|}{ End-Dec. 2010} & \multicolumn{2}{|c|}{ End-March 2011} \\
\hline & Prog. & Est & Prog. & Est. & Prog. & Prel. \\
\hline \multicolumn{7}{|l|}{ Performance Criteria } \\
\hline Basic Non-oil primary fiscal balance (floor) & -363 & -350 & -663 & -658 & -189 & -188 \\
\hline $\begin{array}{l}\text { New medium or long-term nonconcessional external debt } \\
\text { (including leasing) contracted or guaranteed by the government } \\
\text { (ceiling) }{ }^{1,2,3,4}\end{array}$ & 0 & 0 & 0 & 0 & 0 & 0 \\
\hline \multicolumn{7}{|l|}{ Memorandum items } \\
\hline Oil revenue & 1,060 & 910 & 1,949 & 1,758 & 595 & 544 \\
\hline Non-oil primary revenue & 211 & 227 & 465 & 463 & 137 & 137 \\
\hline $\begin{array}{l}\text { Net domestic financing of the government } \\
\text { (ceiling, indicative target) }\end{array}$ & -618 & -188 & -668 & -289 & -383 & -101 \\
\hline \multicolumn{7}{|c|}{$\begin{array}{l}{ }^{1} \text { Excluding rescheduling arrangements and disbursements from the IMF; the minimum grant element is set to } 35 \text { percent. } \\
{ }^{2} \text { Continuous. } \\
{ }^{3} \text { The zero ceiling on nonconcessional external debt does not apply to forthcoming external loans from the European Investment Bank and the } \\
\text { Central African States Development Bank, as specified in paragraph } 11 \text { of the Technical Memorandum of Understanding. } \\
{ }^{4} \text { The zero ceiling also applies to new (i) external debt with an original maturity of less than one year, (ii) oil-collateralized external debt } \\
\text { contracted by or on behalf of the central government, (iii) nonconcessional external debt with a maturity of more than } 1 \text { year contracted or } \\
\text { guaranteed by SNPC, (iv) external arrears on nonreschedulable debt, and (v) to new domestic arrears. }\end{array}$} \\
\hline
\end{tabular}


- Over the first two years of the program, the cumulative adjustment of the basic non-oil primary deficit (BNOPD) was broadly in line with the envisioned adjustment over the course of the whole three year ECF supported arrangement (Figure 3).

\section{The authorities observed the two structural}

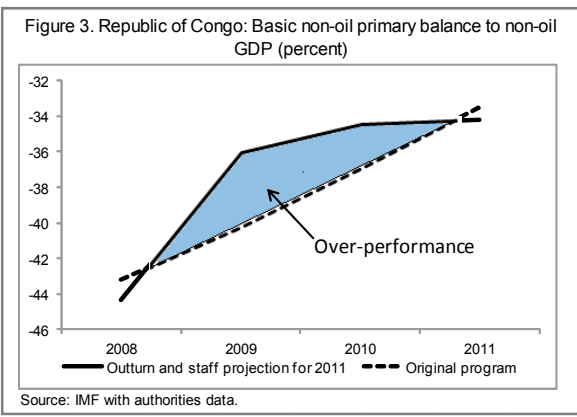

benchmarks aimed at improving oil governance, but encountered early difficulties with the new procurement code, observing this benchmark with delay.

- Oil governance: The authorities have posted the oil certification reports for September and December 2010 on the ministry of economy, finance and budget website, and repatriated the proceeds of oil shipments to the Treasury within 45 days of shipment. Moreover, in December 2010 the EITI Board concluded that Congo had made "meaningful progress" in implementing EITI. ${ }^{1}$

- Procurement: The structural benchmark requires that 80 percent of all contracts over $\$ 500,000$ be subject to competitive bidding. Significant progress was made and the authorities brought various contracts in well below the expected cost. However, as can be expected, the transition was not without challenges, as line ministries have only recently begun to overcome significant teething problems and capacity constraints in implementing the new procedures and previously signed and ongoing contracts have to be brought under the new code. With 2011 annual procurement plans in place, by end-May the authorities had achieved a rate of about 90 percent.

\section{The macroeconomic outlook is favorable, with the increasing stock of basic} infrastructure likely to accelerate poverty-reducing non-oil growth. Oil production in currently operational fields is expected to peak in 2012, but new fields could come on stream starting in 2015, following ongoing negotiations to change the oil taxation regime (Figure 4). Non-oil growth will gain momentum as efforts to boost the business climate bear fruit and completion of public investment projects eases transport and supply bottlenecks. Strong oil prices and prudent policies will further strengthen the external position as external liabilities decline and gross official foreign assets rise to above 100 percent of GDP in 2013. A debt sustainability analysis (DSA, Informational Annex) indicates Congo is at low risk of debt distress and debt dynamics are robust to all standard shocks, but as is the case for the economy as a whole are highly sensitive to volatile international oil prices. Projected large asset accumulation over the longer term eliminates any external borrowing requirement.

\footnotetext{
${ }^{1}$ Extractive Industries Transparency Initiative. Congo’s second Validation Report covers 2007, 2008 and 2009.
} 
Figure 4. Republic of Congo: Oil production profile and oil-dependence

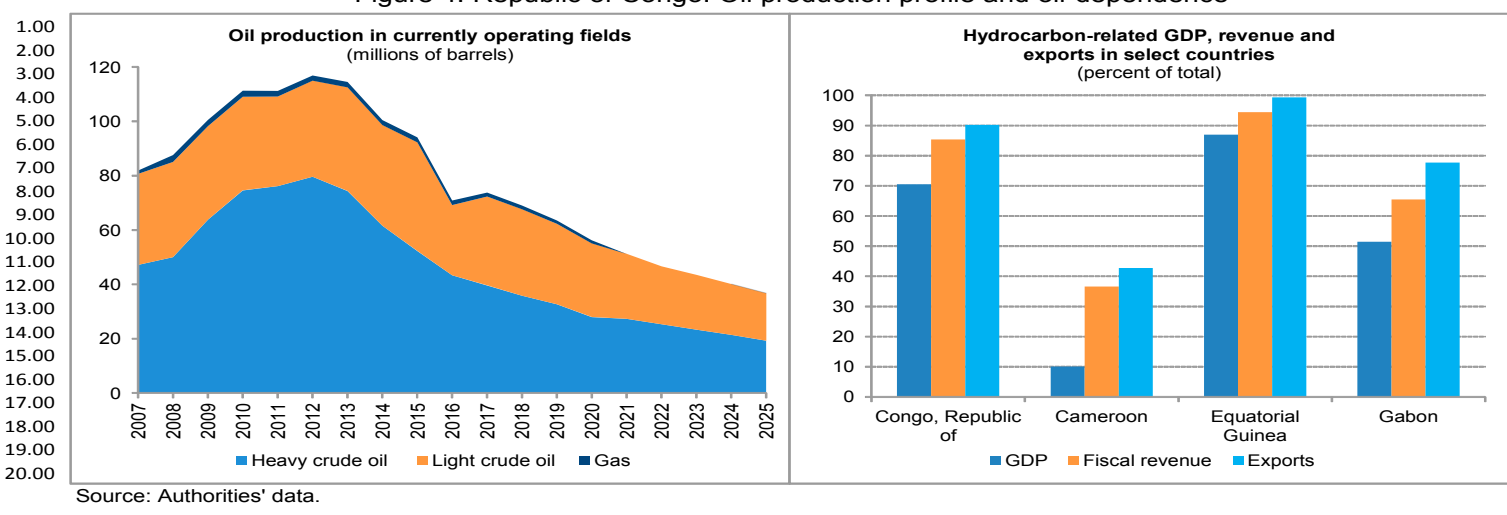

8. With a final test date of end-March 2011, program risks are limited, but looking ahead spending pressures may mount as oil prices climb. Clamors for further scaling up social spending and outlays on fuel subsidies may intensify, but so far, the impact of rising global food and fuel prices is being mitigated by good harvests, improved transportation and some administered prices.

\section{Policy Discussions}

The need for sustained consolidation and the already-large increase in investment spending slated for 2011 call for saving the bulk of the oil windfall. At the same time, macroeconomic stability and structural reforms to date have laid the foundation for taking policy making to the next level. The dialogue focused on the authorities' efforts to strengthen tax policy and fiscal institutions. Efforts aimed at improving the business climate were also discussed.

\section{A. Fiscal policy_Sustained consolidation while making the most of investment}

9. Fiscal policy in 2011 focuses on narrowing the infrastructure gap, while continuing to save a sizable share of oil revenue. To this end, the authorities' BNOPD projection of CFAF750 billion at end-December 2011 (-34.4 percent of non-oil GDP) allows for a 30 percent increase in domestically financed public investment. At current oil price projections, the government will save about 60 percent of total oil revenue ( 22 percent of GDP). Given the need for fiscal consolidation and the already-large increase in public investment, the authorities plan to save the bulk of any future oil windfall.

10. In order to create space for higher capital spending on basic infrastructure including roads and utilities, the authorities are taking the measures contained in the 2011 budget to increase non-oil revenue and restrain current expenditure.

- Revenue: Measures are in train to (a) broaden the tax base (single window for tax payments of forestry and telecoms royalties to the treasury; taxation of transactions related to public contracts); (b) reduce exemptions; and (c) improve tax administration, with particular emphasis on customs appraisal and control. Preliminary data through April indicate that the authorities are on track to exceed the budgeted 2 percentage point of non-oil GDP increase in tax revenue collection, with upside potential if further 
measures are adopted. However, customs revenue will fall short if negotiations with the oil sector fail to accord the minimum 5 percent customs duty envisioned in the budget.

- $\quad$ Current expenditure: Measures aim to bring transfers and spending on materials and supplies down from the high levels experienced in 2010 due to one-off events, and curtail non-essential official travel. The authorities do not intend to raise domestic fuel prices, but have retooled the refinery to increase efficiency and raise domestic supply. ${ }^{2}$ Staff welcomed the expenditure restraint and efforts to boost efficiency at the refinery, but advised that the implicit subsidy resulting from not adjusting domestic prices should be transparently presented in the fiscal accounts.

\section{In order to safeguard investment quality, the authorities are building on} progress to date in public financial management (PFM). Guided by their December 2010 self assessment, they have developed and are beginning to implement a second-generation action plan covering 2011-13 (PAAGIP II) to strengthen investment efficiency. With the procurement code firmly in place, reforms focus on two areas: (a) improving upstream project selection and prioritization and (b) strengthening project implementation. More broadly, the authorities have made progress in strengthening the first three stages of the expenditure chain (i.e., commitment, liquidation, payment order). Staff welcomed the progress to date and the action plan, but noted that a key bottleneck to improvement is the severe weaknesses in the area of treasury operations and control. Staff also encouraged the authorities to revisit the projects slated to begin over the remainder of the current year given the setbacks that were incurred in preparation of the 2011 budget.

\section{B. Reforming the tax system and strengthening fiscal institutions}

\section{The authorities' noted that the tax system is} not reaching its potential, even when compared to other countries in the sub-region. This is consistent with the findings of a 2009 FAD technical assistance (TA) mission, which identified deficiencies in fiscal institutions within a tax system which is unnecessarily complex. ${ }^{3}$ Tax evasion and avoidance are endemicoften aided by reduced rates for certain sectors (i.e., forestry, agricultural inputs) - while VAT and excise exemptions for the oil industry significantly reduce

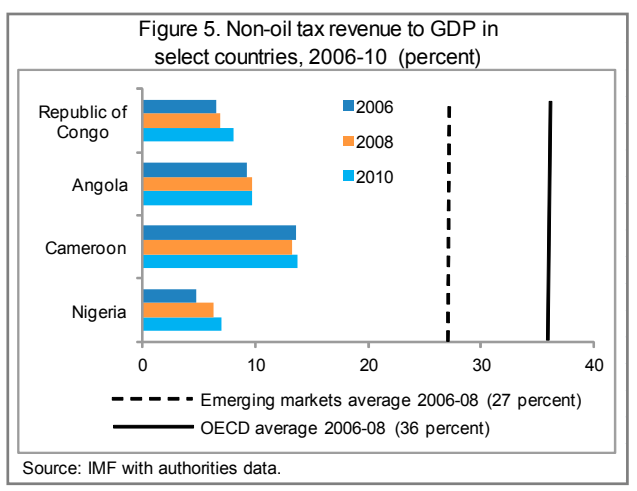
revenue potential.

\footnotetext{
${ }^{2}$ Domestic fuel prices were last adjusted in September 2008, when oil prices were above $\$ 100$ per barrel. At current oil price projections, the authorities estimate an implicit subsidy (refinery losses) of 0.6 percent of GDP.

${ }^{3}$ Characteristics include: high marginal tax rates on capital and labor; a narrow tax base; ubiquitous exemptions often granted at the ministry level; and a distortionary investment system compared with regional peers. Low non-oil revenue collection has mostly resulted from exemptions and tax avoidance.
} 
13. In June 2010 the authorities launched a fiscal reform aimed at improving the design of the tax system and strengthening fiscal institutions, while raising revenue collection. The reform program for 2011-13 is based on the initial report of the Steering Committee and draws heavily on IMF TA recommendations, including those of a February 2011 FAD follow up mission (Box 1). The authorities have (a) conducted a survey of unauthorized fees charged by line ministries (parafiscalité), (b) unified the tax rate and simplified the tax regime for businesses with turnover less than CFAF 40 million (about $\$ 80,000$ ), (c) implemented a unique taxpayer identification system, (d) are in the process of producing a tax expenditure document which will be submitted to parliament along with the 2012 draft budget law, and (e) have eliminated all exemptions not governed by international conventions, the general tax code and the investment code, although the latter is not always respected. The ministry of finance (MOF) is in the process of being reorganized by tax payer units, and computer systems linking all budget operations should be operational by end2011. Ad hoc measures are also being taken to strengthen capacity, reduce tax evasion, improve taxpayer education and ease the cost of compliance. ${ }^{4}$

\section{There was broad agreement that bolder reforms were needed, but these require} increased political consensus. While recognizing the tradeoffs between improving tax competitiveness and increasing revenue collection, the authorities noted that vested interests and key sectors are resisting more sweeping reforms. They have struggled to achieve compliance of line ministries with the prohibition on ad hoc exemptions and fees, and face an uphill battle in garnering political support for eliminating remaining exemptions, including for the important oil sector, and the reduced VAT on the forestry sector. In order to create a space for dialogue and address the impasse, they have created six subcommittees comprised of both the public and private sectors. ${ }^{5}$ Staff encouraged the authorities to strengthen the MOF as an institution, including by amending the organic budget law to make the MOF the only institution charged with putting forward tax policy measures in the context of the draft budget law; to produce and submit to parliament an annual tax expenditure document; and to improve oil revenue projections through increasing transparency regarding the financial position of the national oil company. Staff also encouraged the authorities to use their position as chair of the CEMAC Council of Ministers to push for improvements in the regional excise and tariff system.

\footnotetext{
${ }^{4}$ Measures include: increase tax payer education activities; distribute personal income tax forms directly to employees; implement the newly drafted procedural manual for inspectors on how to conduct an audit; and distribute and enforce the new manual identifying the responsibilities of public sector employees. The hours of operation of the tax department have also been increased, and bonuses are being paid to collection officers.

${ }^{5}$ Subcommittees cover the topics of: streamlining and harmonization of tax rates (direct taxes); VAT and indirect taxation; property and land taxation; parafiscalité; oil sector taxation; and Special Economic Zones.
} 


\section{Box 1. Fiscal reform-Recommendations and actions planned for 2011}

A February 2011 technical assistance mission from the Fiscal Affairs Department (FAD) of the IMF conducted a follow up mission on tax policy. The wide-ranging recommendations are best carried out in the context of a comprehensive three year plan. However, previously identified measures require no further study and can be implemented now.

\section{FAD TA recommendations for 2011 requiring no further study}

\section{$\underline{\text { Tax Policy }}$}

$>$ Reduce distortions and opportunities for tax avoidance: unify the minimum tax rate for all sectors; eliminate the reduced VAT rate on forestry products; and eliminate VAT exemptions on inputs to final products (e.g. agriculture products);

$>$ Simplify the customs clearance procedure to reduce the number of payments by importers;

$>$ Streamline tax incentives and eliminate remaining exemptions, which are mostly related to public enterprises, real estate properties, forestry, alcohol and tobacco prior to implementing further tax rate cuts; and

$>$ Eliminate fees or parafiscalité wherever it acts as an additional tax, rather than a price for a service.

Fiscal Institution Building

$>$ Prepare and present to parliament an annual tax expenditure document to increase transparency regarding the cost of the current tax system; provide a basis for analysis and discussion of tax proposals and tax reform measures; and enhance the legitimacy of the ministry of finance (MOF);

$>$ Include in the 2012 budget law a provision to make the MOF the only entity charged with putting forward fiscal policy measures to parliament as a first step to eventually amending the organic budget law. Proposals of other agencies would pass through a filter at the MOF, who would bear ultimate responsibility, and all proposals would transparently be put forth in the budget document; and

$>$ Increase the leadership role (versus oversight) of the steering committee on fiscal reform.

\section{Authorities' planned actions for 2011}

$>$ Broaden the tax base by extending excises taxes to domestic sales of alcohol and tobacco products (sin taxes) and luxury goods (proposal - in the context of the CEMAC common external tariff);

$>$ Submit to CEMAC a proposal to zero rate capital imports under the common external tariff in order to encourage investment, while taxing companies on their profits;

$>$ Simplify taxation of the informal sector through a single flat turnover tax; and

$>$ Work to define the minimum tax that all businesses should pay to ensure that all companies make some contribution to the government (effective only in 2012). 


\section{Stepping up efforts to improve the business climate}

\section{In February 2011 the Council of Ministers adopted a comprehensive action plan} to improve the business climate. The ultimate aim of the plan is to foster economic diversification and employment creation which will help raise the population out of poverty. The authorities noted that with the support of development partners, six topical working groups have been formed to study and implement measures aimed at achieving the plan's ten objectives, which range from improving the fiscal and legal environment to supporting SMEs, improving access to credit and developing industrial zones. They noted that a framework is now in place for public-private dialogue, and with the help of the European Union progress has already been made in the area of trade and commerce, where a one-stop shop for business registration has been set up; consumer protection and competition laws have been drafted; arbitration manuals have been drawn up; and training is taking place throughout the country for judges and lawyers on OHADA law. Work is also gearing up to establish an SME promotion body, and they are currently considering various modalities to improve access to financing. ${ }^{6}$ All of these reforms will complement ongoing efforts to close the infrastructure gap over the medium term - a first section of the road from the port city to the capital will be completed this year; two sizable electricity generation plants came on stream in 2010, with work on distribution underway; and a new potable water facility is scheduled for completion in 2012.

\section{Staff welcomed these initiatives and encouraged the authorities to push forward} with them in the context of the broader reform agenda, given the important synergies that exist between the planned actions and ongoing work to improve basic infrastructure and reform customs. Various commission reports are currently under consideration by government. In this context, there was broad agreement that increased coordination and alignment of views among the multiple ministries, donors and consultants would be necessary to keep the reform agenda moving ahead. At the same time, staff encouraged the authorities to exercise caution in considering use of a guarantee fund for lending to SMEs given the potentially large fiscal costs associated with such a scheme.

\section{OTHER ISSUES}

\section{The authorities noted that they will adopt the functional classification of pro-} poor spending once the Poverty Reduction Committee has validated the methodology. ${ }^{7}$ This is expected in the coming months. Staff welcomed the improved monitoring that this

\footnotetext{
${ }^{6}$ Recently, credit to SMEs has been supported by a loan from the Arab Bank for Development of Africa. The authorities are also considering other modalities, including establishing a guarantee fund.

${ }^{7}$ The functional classification under consideration was developed in collaboration with AFRITAC and yields higher estimates of pro-poor spending than the authorities' current classification.
} 
could entail, but stressed that it was even more important to ensure that budgeted social funds are not only made available when requested but also used effectively. Strengthening treasury operations and expenditure monitoring of ministries in charge of social spending is vital to improving public service provision. The authorities should work with the development partners to strengthen capacity.

\section{The authorities stressed their commitment to work toward resolving outstanding} debt issues. Agreements have been signed with the vast majority creditors. Discussions with the outstanding non-Paris Club bilateral creditor will resume at the next Joint Commission meeting scheduled for 2012, and remaining bilateral institutions would need to respond to the Congolese offer. Regarding commercial creditors, cases with two litigating creditors are ongoing in the courts, while discussions to obtain comparable treatment with one creditor continue.

\section{Staff Appraisal}

19. The near-term outlook is favorable. Strong policies and improving external conditions will support macroeconomic stability, while fiscal consolidation in excess of original program targets and progress on key structural reforms have built resilience to shocks.

20. Sustained efforts to strengthen policies and the scaling up of investment in basic infrastructure bode well for a take-off in non-oil growth and durable poverty reduction. Given the already-large increase in investment and the need for fiscal consolidation over the medium term, the bulk of the oil windfall should be saved. Ensuring that pro-poor spending reaches its intended target groups may help quell clamors for higher spending - the main risk going forward.

21. Structural reforms have moved forward, particularly in the areas of PFM and oil wealth management. Especially notable in this regard is the implementation of the new procurement code which requires competitive bidding. The observance with delay of the structural benchmark containing an eighty percent threshold for larger projects reflects transitional challenges faced by line ministries and the need to bring ongoing projects under the code. That said, much remains to be done to address the severe weaknesses in treasury operations and control, and efforts to strengthen project selection and implementation should continue.

22. In the period ahead, increasing focus should be placed on strengthening policy making and building institutions, while continuing to work toward improving transparency and governance of oil wealth. Reforms underway in the fiscal area will help reduce the complexity of the tax system, improve efficiency and reduce tax avoidance and evasion. The ministry of finance should be strengthened, including by making it the sole institution responsible for putting forth tax policy measures in the context of the draft budget law. This should be complemented by annual production and submission of a tax expenditure document which presents the cost of current and proposed policies, which will help garner 
the needed support for deeper tax measures encountering resistance from vested interests. At the same time, increasing transparency regarding the financial position of the national oil company through an annual published audit would go a long way toward better understanding the consistent underperformance of fiscal oil revenues relative to projections.

23. The welcome initiatives to improve the business climate should continue in the context of the broader reform agenda to increase basic infrastructure and improve customs. Increased coordination and alignment among the multiple ministries, donors and consultants will be necessary to keep the reform agenda moving ahead.

24. Reaching the completion point has significantly reduced Congo's debt burden. Best efforts to conclude bilateral agreements as soon as possible and good faith efforts to obtain comparable treatment from all remaining commercial creditors should continue. New debt should only be considered if extended on highly concessional terms.

25. Staff recommends completion of the fifth and sixth reviews under the ECF arrangement and disbursement of the sixth and seventh loans, in an amount equivalent to SDR 1,208,570 and SDR 1,208,580, respectively. Staff also supports the authorities' request to rephrase the seventh disbursement to end-July to better align it with completion of the sixth and final review. 
Table 1. Republic of Congo: Selected Economic and Financial Indicators, 2008-13

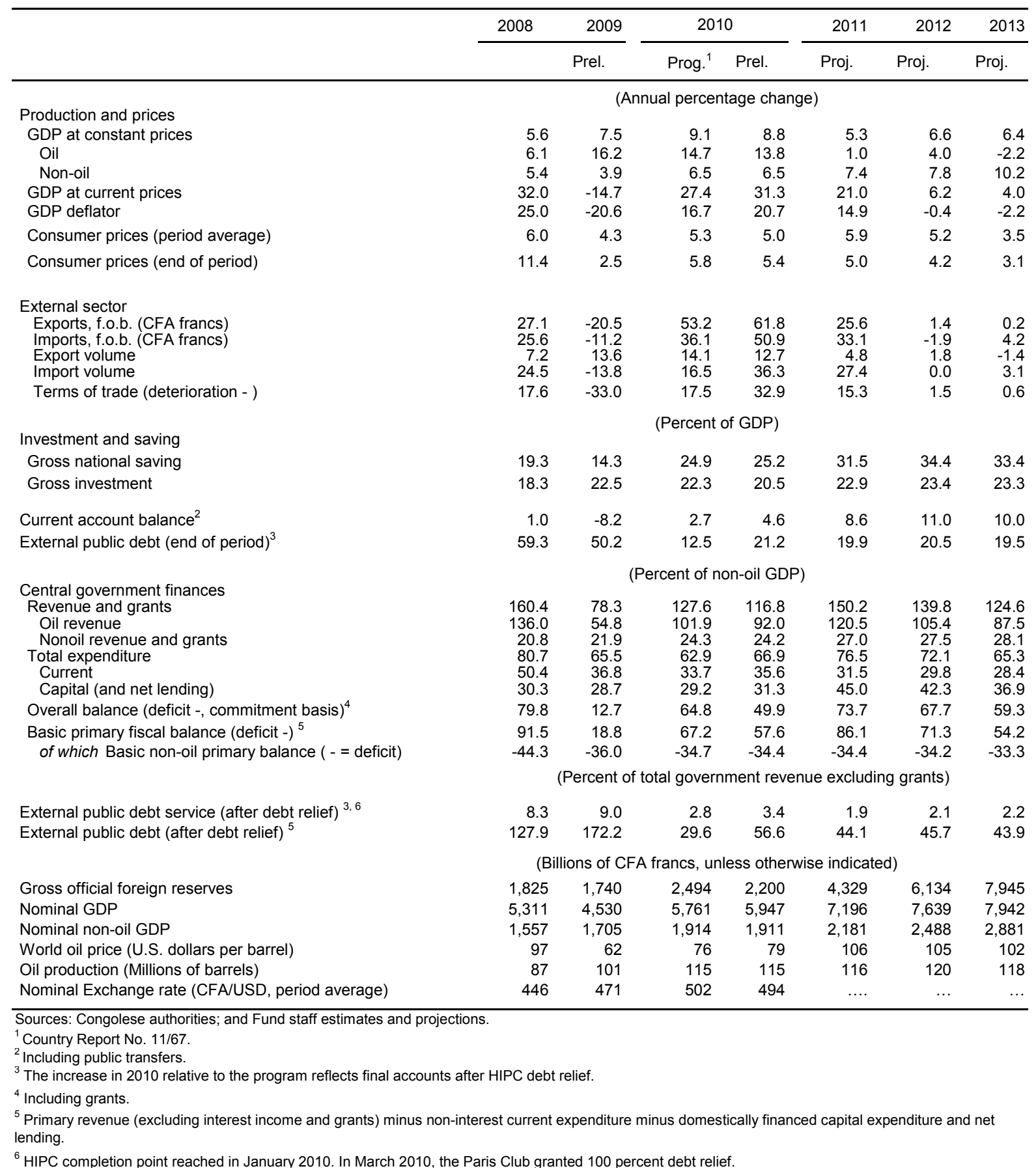


Table 2. Republic of Congo, Medium Term Balance of Payments, 2008-13

\begin{tabular}{|c|c|c|c|c|c|c|c|}
\hline & \multirow[t]{2}{*}{2008} & \multirow{2}{*}{$\begin{array}{r}2009 \\
\text { Est. }\end{array}$} & \multicolumn{2}{|c|}{2010} & \multirow{2}{*}{$\begin{array}{c}2011 \\
\text { Proj. }\end{array}$} & \multirow{2}{*}{$\begin{array}{c}2012 \\
\text { Proj. }\end{array}$} & \multirow{2}{*}{$\begin{array}{r}2013 \\
\text { Proj }\end{array}$} \\
\hline & & & Prog. ${ }^{1}$ & Prel. & & & \\
\hline & \multicolumn{7}{|c|}{ (Billions of CFA francs) } \\
\hline Current account & 53 & -371 & 155 & 275 & 620 & 840 & 798 \\
\hline Trade balance & 2,448 & 1,825 & 3,055 & 3,078 & 3,733 & 3,860 & 3,776 \\
\hline Exports, f.o.b. & 3,749 & 2,980 & 4,633 & 4,821 & 6,053 & 6,137 & 6,148 \\
\hline Oil sector & 3,386 & 2,657 & 4,110 & 4,282 & 5,356 & 5,377 & 5,287 \\
\hline Non-oil sector & 363 & 323 & 523 & 539 & 697 & 759 & 862 \\
\hline Imports, f.o.b. & $-1,301$ & $-1,155$ & $-1,578$ & $-1,743$ & $-2,320$ & $-2,276$ & $-2,372$ \\
\hline Oil sector & -307 & -383 & -405 & -513 & -586 & -591 & -591 \\
\hline Government & -293 & -259 & -470 & -511 & -784 & -675 & -682 \\
\hline Non-oil private sector & -701 & -513 & -704 & -718 & -949 & $-1,010$ & $-1,098$ \\
\hline Balance of services & $-1,014$ & -943 & $-1,089$ & $-1,293$ & $-1,281$ & $-1,291$ & $-1,326$ \\
\hline Oil sector & -739 & -737 & -784 & -978 & -911 & -918 & -920 \\
\hline Nonoil sector & -275 & -206 & -305 & -315 & -370 & -373 & -406 \\
\hline Income & $-1,385$ & $-1,234$ & $-1,784$ & $-1,477$ & $-1,829$ & $-1,745$ & $-1,667$ \\
\hline Labor income & -46 & -36 & -58 & -60 & -76 & -76 & -75 \\
\hline Investment income & $-1,339$ & $-1,198$ & $-1,726$ & $-1,417$ & $-1,753$ & $-1,669$ & $-1,592$ \\
\hline Current transfers (net) & 5 & -19 & -28 & -33 & -3 & 16 & 14 \\
\hline Capital account & 35 & 15 & 1,288 & 1,318 & 35 & 57 & 60 \\
\hline Debt forgiveness ${ }^{2}$ & 0 & 0 & 1,278 & 1,313 & 0 & 0 & 0 \\
\hline Official grants & 35 & 15 & 10 & 4 & 35 & 57 & 60 \\
\hline Other & 0 & 0 & 0 & 0 & 0 & 0 & 0 \\
\hline Financial account & 1,031 & 161 & -695 & $-1,240$ & 1,507 & 909 & 956 \\
\hline Direct investment (net) & 1,131 & 879 & 1,093 & 1,094 & 1,383 & 1,402 & 1,435 \\
\hline Of which: oil sector & 1,009 & 834 & 951 & 991 & 1,261 & 1,259 & 1,264 \\
\hline Portfolio investment & -1 & 0 & 0 & 0 & 0 & 0 & 0 \\
\hline Other investment & -99 & -719 & $-1,788$ & $-2,334$ & 125 & -493 & -479 \\
\hline Medium and long term & -161 & -79 & $-1,290$ & $-1,277$ & 173 & 145 & -22 \\
\hline Public sector & -82 & -80 & $-1,301$ & $-1,284$ & 205 & 143 & -9 \\
\hline Drawings & 47 & 6 & 36 & 80 & 256 & 202 & 54 \\
\hline Project & 46 & 6 & 36 & 80 & 256 & 202 & 54 \\
\hline Program & 1 & 0 & 0 & 0 & 0 & 0 & 0 \\
\hline Amortization $^{2}$ & -129 & -86 & $-1,337$ & $-1,363$ & -52 & -59 & -63 \\
\hline Private sector & -79 & 1 & 11 & 7 & -31 & 3 & -13 \\
\hline Oil & -76 & 8 & 9 & 10 & 30 & 35 & 35 \\
\hline Non-oil & -3 & -7 & 2 & -3 & -61 & -32 & -48 \\
\hline Short term & 61 & -640 & -498 & $-1,058$ & -49 & -639 & -457 \\
\hline Errors and omissions & 0 & -2 & 0 & 0 & 0 & 0 & 0 \\
\hline Overall balance of payments & 1,120 & -196 & 748 & 353 & 2,162 & 1,806 & 1,813 \\
\hline Financing & $-1,120$ & 196 & -748 & -353 & $-2,162$ & $-1,806$ & $-1,813$ \\
\hline Reserve financing & -850 & 132 & -760 & -406 & $-2,128$ & $-1,806$ & $-1,813$ \\
\hline IMF (net) ${ }^{3}$ & 1 & 2 & -6 & -6 & 2 & -1 & -2 \\
\hline Purchases / Disbursments & 1 & 2 & 2 & 2 & 2 & 0 & 0 \\
\hline Repurchases / Repayments & 0 & 0 & 0 & 0 & 0 & -1 & -2 \\
\hline Other reserves & -850 & 132 & -753 & -406 & $-2,128$ & $-1,806$ & $-1,813$ \\
\hline Exceptional financing ${ }^{4}$ & -270 & 64 & 12 & 53 & -34 & 0 & 0 \\
\hline Net change in arrears & -801 & -15 & -402 & -64 & -34 & 0 & 0 \\
\hline Debt cancellation & 207 & 58 & 18 & 91 & 0 & 0 & 0 \\
\hline Debt rescheduling & 325 & 21 & 396 & 26 & 0 & 0 & 0 \\
\hline Financing gap (- = surplus) & 0 & 0 & 0 & 0 & 0 & 0 & 0 \\
\hline
\end{tabular}

Sources: BEAC; and Fund staff estimates and projections.

${ }^{1}$ Country Report No. 11/67.

${ }^{2}$ Includes stock debt relief of the HIPC completion point.

${ }^{3}$ Includes assumed disbursements under the new PRGF.

${ }^{4}$ Includes flow debt relief from Paris Club and London Club, and payments to litigating creditors. 
Table 3a. Republic of Congo: Central Government Operations, 2008-13

\begin{tabular}{|c|c|c|c|c|c|c|c|}
\hline & \multirow[t]{2}{*}{2008} & \multirow[t]{2}{*}{2009} & \multicolumn{2}{|c|}{2010} & \multirow{2}{*}{$\begin{array}{l}2011 \\
\text { Proj. }\end{array}$} & \multirow{2}{*}{$\begin{array}{l}2012 \\
\text { Proj. }\end{array}$} & \multirow{2}{*}{$\begin{array}{l}2013 \\
\text { Proj. }\end{array}$} \\
\hline & & & Prog. ${ }^{1}$ & Prel. & & & \\
\hline & \multicolumn{7}{|c|}{ (Billions of CFA francs) } \\
\hline Revenue and grants & 2,497 & 1,335 & 2,443 & 2,231 & 3,274 & 3,480 & 3,589 \\
\hline Primary Revenue & 2,442 & 1,307 & 2,414 & 2,222 & 3,203 & 3,296 & 3,314 \\
\hline Oil revenue & 2,118 & 934 & 1,949 & 1,758 & 2,627 & 2,624 & 2,521 \\
\hline Non-oil revenue & 324 & 373 & 465 & 463 & 576 & 672 & 794 \\
\hline Investment income & 20 & 13 & 18 & 6 & 36 & 127 & 215 \\
\hline Grants & 35 & 15 & 10 & 4 & 35 & 57 & 60 \\
\hline Expenditure and net lending & 1,256 & 1,118 & 1,203 & 1,278 & 1,668 & 1,795 & 1,881 \\
\hline of which primary expenditure & 1,024 & 1,023 & 1,145 & 1,136 & 1,366 & 1,523 & 1,753 \\
\hline Current expenditure & 785 & 627 & 644 & 680 & 687 & 742 & 817 \\
\hline Wages & 166 & 175 & 180 & 180 & 225 & 255 & 293 \\
\hline Other current expenditure & 446 & 355 & 409 & 399 & 411 & 432 & 466 \\
\hline Local authorities & 23 & 24 & 43 & 43 & 40 & 42 & 44 \\
\hline Interest & 150 & 73 & 12 & 59 & 11 & 13 & 14 \\
\hline Domestic & 10 & 9 & 0 & 1 & 0 & 0 & 0 \\
\hline External & 140 & 64 & 12 & 57 & 11 & 13 & 14 \\
\hline Capital expenditure & 471 & 490 & 559 & 598 & 981 & 1,053 & 1,064 \\
\hline Domestically financed & 390 & 469 & 513 & 515 & 690 & 794 & 950 \\
\hline o.w. HIPC financed & 7 & 37 & 17 & 14 & 40 & 0 & 0 \\
\hline Externally financed & 81 & 21 & 46 & 84 & 291 & 259 & 114 \\
\hline Net lending & 0 & 1 & 0 & 0 & 0 & 0 & 0 \\
\hline Basic primary balance $^{2}$ & 1,424 & 320 & 1,286 & 1,101 & 1,877 & 1,773 & 1,561 \\
\hline Of which: Basic non-oil primary balance & -690 & -614 & -663 & -658 & -750 & -851 & -959 \\
\hline \multicolumn{8}{|l|}{ Balance, commitment basis } \\
\hline Excluding grants & 1,206 & 202 & 1,229 & 949 & 1,571 & 1,628 & 1,648 \\
\hline Including grants & 1,242 & 216 & 1,239 & 953 & 1,606 & 1,685 & 1,708 \\
\hline Of which: Non-oil balance & -876 & -718 & -738 & -805 & $-1,021$ & -939 & -812 \\
\hline Change in arrears & -944 & -124 & -371 & -225 & -38 & -44 & -33 \\
\hline External & -801 & 0 & -222 & -64 & 0 & 0 & 0 \\
\hline Domestic & -143 & -124 & -149 & -161 & -38 & -44 & -33 \\
\hline Balance, cash basis & 298 & 92 & 868 & 728 & 1,568 & 1,641 & 1,675 \\
\hline Financing & -298 & -92 & -868 & -728 & $-1,568$ & $-1,641$ & $-1,675$ \\
\hline Foreign (net) & 408 & -34 & -14 & -366 & 647 & 151 & -2 \\
\hline Drawings & 47 & 6 & 36 & 80 & 256 & 202 & 54 \\
\hline Amortization due & -170 & -106 & -68 & -64 & -43 & -51 & -56 \\
\hline Rescheduling obtained & 325 & 21 & 0 & 26 & 0 & 0 & 0 \\
\hline Debt cancellation & 207 & 52 & 18 & 56 & 0 & 0 & 0 \\
\hline Exceptional assistance (including debt relief) ${ }^{3}$ & 0 & -2 & 0 & 0 & 0 & 0 & 0 \\
\hline Other & 0 & 0 & -200 & -464 & 434 & 0 & 0 \\
\hline Domestic (net) & -706 & -59 & -655 & -362 & $-2,214$ & $-1,792$ & $-1,673$ \\
\hline Banking system (net) & -599 & 122 & -668 & -289 & $-2,253$ & $-1,792$ & $-1,673$ \\
\hline Nonbank financing & -107 & -181 & 13 & -73 & 38 & 0 & 0 \\
\hline Of which, statistical float & -82 & -107 & 0 & -18 & 0 & 0 & 0 \\
\hline Financing gap (- = surplus) & 0 & 0 & 0 & 0 & 0 & 0 & 0 \\
\hline \multicolumn{8}{|l|}{ Memorandum items: } \\
\hline GDP at current market prices & 5,311 & 4,530 & 5,761 & 5,947 & 7,196 & 7,639 & 7,942 \\
\hline Non-oil GDP at market prices & 1,557 & 1,705 & 1,914 & 1,911 & 2,181 & 2,488 & 2,881 \\
\hline Pro poor spending & 342 & 401 & 465 & 465 & 567 & 682 & 792 \\
\hline
\end{tabular}

Sources: Congolese authorities; and Fund staff estimates and projections.

${ }^{1}$ Country Report No. 11/67.

${ }^{2}$ Primary revenue (excluding interest income and grants) minus noninterest current expenditure minus domestically finance capital expenditure (excluding HIPC-financed capital expenditure) and net lending.

${ }^{3}$ HIPC completion point reached in January 2010. In March 2010, the Paris Club granted 100 percent debt relief. 
Table 3b. Republic of Congo: Central Government Operations, 2008-13

\begin{tabular}{|c|c|c|c|c|c|c|c|}
\hline & \multirow[t]{2}{*}{2008} & \multirow[t]{2}{*}{2009} & \multicolumn{2}{|c|}{2010} & \multirow{2}{*}{$\frac{2011}{\text { Proj. }}$} & \multirow{2}{*}{$\begin{array}{c}2012 \\
\text { Proj. }\end{array}$} & \multirow{2}{*}{$\begin{array}{l}2013 \\
\text { Proj. }\end{array}$} \\
\hline & & & Prog. ${ }^{1}$ & Prel. & & & \\
\hline & \multicolumn{7}{|c|}{ (Percent of non-oil GDP) } \\
\hline Revenue and grants & 160.4 & 78.3 & 127.6 & 116.8 & 150.2 & 139.8 & 124.6 \\
\hline Primary Revenue & 156.8 & 76.7 & 126.2 & 116.2 & 146.9 & 132.5 & 115.0 \\
\hline Oil revenue & 136.0 & 54.8 & 101.9 & 92.0 & 120.5 & 105.4 & 87.5 \\
\hline Non-oil revenue & 20.8 & 21.9 & 24.3 & 24.2 & 26.4 & 27.0 & 27.5 \\
\hline Investment income & 1.3 & 0.8 & 0.9 & 0.3 & 1.7 & 5.1 & 7.5 \\
\hline Grants & 2.3 & 0.9 & 0.5 & 0.2 & 1.6 & 2.3 & 2.1 \\
\hline $\begin{array}{l}\text { Expenditure and net lending } \\
\text { of which primary expenditure }\end{array}$ & $\begin{array}{l}80.7 \\
65.8\end{array}$ & $\begin{array}{l}65.6 \\
600\end{array}$ & $\begin{array}{l}62.9 \\
59.8\end{array}$ & $\begin{array}{l}66.9 \\
59.4\end{array}$ & $\begin{array}{l}76.5 \\
626\end{array}$ & $\begin{array}{l}72.1 \\
612\end{array}$ & $\begin{array}{l}65.3 \\
608\end{array}$ \\
\hline Current expenditure & 50.4 & 36.8 & 33.7 & 35.6 & 31.5 & 29.8 & 28.4 \\
\hline Wages & 10.7 & 10.3 & 9.4 & 9.4 & 10.3 & 10.3 & 10.2 \\
\hline Other current expenditure & 28.6 & 20.8 & 21.4 & 20.9 & 18.8 & 17.4 & 16.2 \\
\hline Local authorities & 1.5 & 1.4 & 2.2 & 2.2 & 1.8 & 1.7 & 1.5 \\
\hline Interest & 9.6 & 4.3 & 0.6 & 3.1 & 0.5 & 0.5 & 0.5 \\
\hline Domestic & 0.6 & 0.6 & 0.0 & 0.1 & 0.0 & 0.0 & 0.0 \\
\hline External & 9.0 & 3.7 & 0.6 & 3.0 & 0.5 & 0.5 & 0.5 \\
\hline Capital expenditure & 30.3 & 28.7 & 29.2 & 31.3 & 45.0 & 42.3 & 36.9 \\
\hline Domestically financed & 25.0 & 27.5 & 26.8 & 26.9 & 31.6 & 31.9 & 33.0 \\
\hline o.w. HIPC financed & 0.4 & 2.2 & 0.9 & 0.8 & 1.8 & 0.0 & 0.0 \\
\hline Externally financed & 5.2 & 1.2 & 2.4 & 4.4 & 13.4 & 10.4 & 4.0 \\
\hline Net lending & 0.0 & 0.1 & 0.0 & 0.0 & 0.0 & 0.0 & 0.0 \\
\hline Basic primary balance $^{2}$ & 91.5 & 18.8 & 67.2 & 57.6 & 86.1 & 71.3 & 54.2 \\
\hline Of which: Basic non-oil primary balance & -44.3 & -36.0 & -34.7 & -34.4 & -34.4 & -34.2 & -33.3 \\
\hline \multicolumn{8}{|l|}{ Balance, commitment basis } \\
\hline Excluding grants & 77.5 & 11.8 & 64.2 & 49.7 & 72.0 & 65.4 & 57.2 \\
\hline Including grants & 79.8 & 12.7 & 64.8 & 49.9 & 73.7 & 67.7 & 59.3 \\
\hline Of which: Non-oil balance & -56.3 & -42.1 & -38.6 & -42.1 & -46.8 & -37.7 & -28.2 \\
\hline Change in arrears & -60.6 & -7.3 & -19.4 & -11.8 & -1.8 & -1.8 & -1.2 \\
\hline External & -51.5 & 0.0 & -11.6 & -3.4 & 0.0 & 0.0 & 0.0 \\
\hline Domestic & -9.2 & -7.3 & -7.8 & -8.4 & -1.8 & -1.8 & -1.2 \\
\hline Balance, cash basis & 19.1 & 5.4 & 45.4 & 38.1 & 71.9 & 66.0 & 58.1 \\
\hline Financing & -19.1 & -5.4 & -45.4 & -38.1 & -71.9 & -66.0 & -58.1 \\
\hline Foreign (net) & 26.2 & -2.0 & -0.7 & -19.1 & 29.7 & 6.1 & -0.1 \\
\hline Drawings & 3.0 & 0.4 & 1.9 & 4.2 & 11.8 & 8.1 & 1.9 \\
\hline Amortization due & -10.9 & -6.2 & -3.6 & -3.3 & -2.0 & -2.0 & -1.9 \\
\hline Rescheduling obtained & 20.9 & 1.2 & 0.0 & 1.4 & 0.0 & 0.0 & 0.0 \\
\hline Debt cancellation & 13.3 & 3.0 & 0.9 & 2.9 & 0.0 & 0.0 & 0.0 \\
\hline Exceptional assistance (including debt relief) ${ }^{3}$ & 0.0 & -0.1 & 0.0 & 0.0 & 0.0 & 0.0 & 0.0 \\
\hline Other & 0.0 & 0.0 & -10.5 & -24.3 & 19.9 & 0.0 & 0.0 \\
\hline Domestic (net) & -45.4 & -3.4 & -34.2 & -18.9 & -101.6 & -72.0 & -58.1 \\
\hline Banking system (net) & -38.5 & 7.2 & -34.9 & -15.1 & -103.3 & -72.0 & -58.1 \\
\hline Nonbank financing & -6.9 & -10.6 & 0.7 & -3.8 & 1.7 & 0.0 & 0.0 \\
\hline Financing gap (- = surplus) & 0.0 & 0.0 & 0.0 & 0.0 & 0.0 & 0.0 & 0.0 \\
\hline Memorandum items: & \multicolumn{7}{|c|}{ (Percent of GDP, unless otherwise indicated) } \\
\hline Revenue and grants & 47.0 & 29.5 & 42.4 & 37.5 & 45.5 & 45.6 & 45.2 \\
\hline Oil revenue & 46.7 & 20.6 & 33.8 & 29.6 & 36.5 & 34.3 & 31.7 \\
\hline Non oil revenue & 6.1 & 8.2 & 8.1 & 7.8 & 8.0 & 8.8 & 10.0 \\
\hline Current expenditure & 11.2 & 13.9 & 11.2 & 11.4 & 9.5 & 9.7 & 10.3 \\
\hline Capital expenditure & 8.9 & 10.8 & 9.7 & 10.1 & 13.6 & 13.8 & 13.4 \\
\hline Basic primary fiscal balance & 26.8 & 7.1 & 22.3 & 18.5 & 26.1 & 23.2 & 19.7 \\
\hline Of which: Basic non oil primary fiscal balance & -13.0 & -13.6 & -11.5 & -11.1 & -10.4 & -11.1 & -12.1 \\
\hline Balance, cash basis & 5.6 & 2.0 & 15.1 & 12.2 & 21.8 & 21.5 & 21.1 \\
\hline Pro poor spending (percent of non-oil GDP) & 21.9 & 23.5 & 24.3 & 24.3 & 26.0 & 27.4 & 27.5 \\
\hline
\end{tabular}

Sources: Congolese authorities; and Fund staff estimates and projections.

${ }^{1}$ Country Report No. 11/67.

${ }^{2}$ Primary revenue (excluding interest income and grants) minus noninterest current expenditure minus domestically financed capital expenditure (excluding HIPC-financed capital expenditure) and net lending

${ }^{3}$ HIPC completion point reached in January 2010. In March 2010, the Paris Club granted 100 percent debt relief. 
Table 4. Republic of Congo: Monetary Survey, 2008-11

\begin{tabular}{|c|c|c|c|c|c|}
\hline & \multirow[t]{2}{*}{2008} & \multirow[t]{2}{*}{2009} & \multicolumn{2}{|c|}{2010} & \multirow{2}{*}{$\frac{2011}{\text { Proj. }}$} \\
\hline & & & Prog. $^{1}$ & Prel. & \\
\hline Monetary survey & \multicolumn{4}{|c|}{ (Billions of CFA francs) } & \\
\hline Net foreign assets & 1,877 & 1,723 & 2,584 & 2,325 & 4,646 \\
\hline Central bank & 1,788 & 1,656 & 2,415 & 2,062 & 4,190 \\
\hline Deposit money banks & 89 & 176 & 169 & 263 & 456 \\
\hline Net domestic assets & -908 & -705 & $-1,441$ & -911 & $-3,032$ \\
\hline Net domestic credit & -893 & -619 & $-1,355$ & -971 & $-3,092$ \\
\hline Net credit to the public sector & $-1,083$ & -962 & $-1,628$ & $-1,314$ & $-3,498$ \\
\hline Net credit to the Government & $-1,079$ & -956 & $-1,624$ & $-1,243$ & $-3,496$ \\
\hline Central bank & $-1,072$ & -956 & $-1,623$ & $-1,305$ & $-3,489$ \\
\hline Credit to the economy & 167 & 218 & 274 & 325 & 384 \\
\hline Other items, net & -15 & -86 & -86 & 60 & 60 \\
\hline Broad money & 970 & 1,019 & 1,143 & 1,415 & 1,614 \\
\hline Currency outside banks & 344 & 342 & 384 & 408 & 466 \\
\hline Demand deposits & 499 & 539 & 605 & 843 & 962 \\
\hline \multirow[t]{2}{*}{ Time deposits } & 126 & 137 & 154 & 163 & 186 \\
\hline & \multicolumn{5}{|c|}{ (Changes in perecent of beginning-of-period broad money) } \\
\hline Net foreign assets & 109.0 & -15.9 & 73.9 & 59.1 & 164.1 \\
\hline Net domestic assets & -72.6 & 21.0 & -61.7 & -20.2 & -150.0 \\
\hline Net domestic credit & -72.2 & 28.3 & -61.7 & -34.6 & -150.0 \\
\hline Net credit to the government & -84.3 & 12.6 & -65.6 & -28.2 & -159.2 \\
\hline \multirow[t]{2}{*}{ Credit to the private sector } & 10.7 & 5.2 & 3.8 & 10.5 & 4.2 \\
\hline & \multicolumn{5}{|c|}{ (Annual percent changes, unless otheriwise indicated) } \\
\hline Broad money & 36.4 & 5.0 & 12.2 & 38.9 & 14.1 \\
\hline Reserve money & 43.3 & -9.6 & 12.2 & 69.9 & 6.9 \\
\hline \multicolumn{6}{|l|}{ Velocity (non-oil GDP/End of period M2) } \\
\hline Non-oil GDP/Average M2 & 2.1 & 2.5 & 2.7 & 2.2 & 2.7 \\
\hline \multirow[t]{2}{*}{ Non-oil GDP/End period M2 } & 1.6 & 1.7 & 2.5 & 1.4 & 1.4 \\
\hline & \multicolumn{4}{|c|}{ (Percent) } & \\
\hline Total GDP growth & 32.0 & -14.7 & 27.4 & 31.3 & 21.0 \\
\hline Non-oil GDP growth & 11.9 & 9.5 & 12.2 & 12.1 & 14.1 \\
\hline Credit to the private sector/Non-oil GDP & 10.7 & 12.8 & 14.3 & 17.0 & 17.6 \\
\hline
\end{tabular}

Sources: BEAC; and Fund staff estimates and projections.

${ }^{1}$ Country Report No. 11/67. 
Table 5. Republic of Congo: Quantitative targets, 2010-11

(Billions of CFA francs; cumulative from January; unless otherwise indicated)

\begin{tabular}{|c|c|c|c|c|c|c|}
\hline & \multicolumn{2}{|c|}{ End-Jun. 2010} & \multicolumn{2}{|c|}{ End-Dec. 2010} & \multicolumn{2}{|c|}{ End-March 2011} \\
\hline & $\begin{array}{l}\text { Perf. } \\
\text { Criteria }\end{array}$ & Est & $\begin{array}{l}\text { Perf. } \\
\text { Criteria }\end{array}$ & Est. & $\begin{array}{l}\text { Perf. } \\
\text { Criteria }\end{array}$ & Prel. \\
\hline \multicolumn{7}{|l|}{ Quantitative targets } \\
\hline Basic Non-oil primary fiscal balance (floor) & -363 & -350 & -663 & -658 & -189 & -188 \\
\hline $\begin{array}{l}\text { New medium or long-term nonconcessional external debt } \\
\text { (including leasing) contracted or guaranteed by the government } \\
\text { (ceiling) }{ }^{1,2,3}\end{array}$ & 0 & 0 & 0 & 0 & 0 & 0 \\
\hline $\begin{array}{l}\text { New external debt (including leasing) with an original maturity of } \\
\text { less than one year (ceiling) }{ }^{2}\end{array}$ & 0 & 0 & 0 & 0 & 0 & 0 \\
\hline $\begin{array}{l}\text { New oil-collateralized external debt contracted by or on behalf of } \\
\text { the central government (ceiling) }{ }^{2}\end{array}$ & 0 & 0 & 0 & 0 & 0 & 0 \\
\hline $\begin{array}{l}\text { New nonconcessional external debt with a maturity of more than } \\
1 \text { year contracted or guaranteed by SNPC (ceiling) }{ }^{2}\end{array}$ & 0 & 0 & 0 & 0 & 0 & 0 \\
\hline New external arrears on nonreschedulable debt (ceiling) ${ }^{2}$ & 0 & 0 & 0 & 0 & 0 & 0 \\
\hline New domestic arrears ${ }^{2}$ & 0 & 0 & 0 & 0 & 0 & 0 \\
\hline \multicolumn{7}{|l|}{ Memorandum items } \\
\hline Oil revenue & 1,060 & 910 & 1,949 & 1,758 & 595 & 544 \\
\hline Non-oil primary revenue & 211 & 227 & 465 & 463 & 137 & 137 \\
\hline $\begin{array}{l}\text { Net domestic financing of the government } \\
\text { (ceiling, indicative target) }\end{array}$ & -618 & -188 & -668 & -289 & -383 & -101 \\
\hline
\end{tabular}

${ }^{1}$ Excluding rescheduling arrangements and disbursements from the IMF; the minimum grant element is set to 35 percent.

${ }^{2}$ Continuous.

${ }^{3}$ The zero ceiling on nonconcessional external debt does not apply to forthcoming external loans from the European Investment Bank and the Central African States Development Bank, as specified in paragraph 11 of the Technical Memorandum of Understanding. 
Table 6. Republic of Congo: Structural Benchmarks under the ECF Arrangement, 2010-11

\begin{tabular}{|c|c|c|c|}
\hline Measures & $\begin{array}{l}\text { Status } \\
\text { End- Dec } \\
2010\end{array}$ & $\begin{array}{l}\text { Status } \\
\text { End-March } \\
2011\end{array}$ & Timing \\
\hline \multicolumn{4}{|l|}{$\begin{array}{l}\text { Public financial management and domestic revenue } \\
\text { mobilization } \\
\text { Justification: Strengthen investment quality }\end{array}$} \\
\hline $\begin{array}{l}\text { Support implementation of the new procurement code by } \\
\text { ensuring that } 80 \text { percent of public contracts having a total } \\
\text { value of over CFAF } 250 \text { million are open to competitive } \\
\text { bidding. }\end{array}$ & $\begin{array}{l}\text { Observed, } \\
\text { with delay }\end{array}$ & $\begin{array}{l}\text { Observed, } \\
\text { with delay }\end{array}$ & Continuous \\
\hline \multicolumn{4}{|l|}{$\begin{array}{l}\text { Governance and natural resource management } \\
\text { Justification: Continue strengthening of oil sector governance }\end{array}$} \\
\hline $\begin{array}{l}\text { Have an internationally reputable audit firm certify oil revenue } \\
\text { quarterly, using the same specifications as for the } 2003 \\
\text { certification and with no restrictions on access to the } \\
\text { information; and publish the certification reports on the } \\
\text { website of the ministry of economy, finance and budget } \\
\text { (www.mefb-cg.org). Also, the government will post on the } \\
\text { website not only the audit but for each report, a note } \\
\text { addressing comments by the auditors. }\end{array}$ & $\begin{array}{l}\text { Observed, } \\
\text { with delay }\end{array}$ & $\begin{array}{l}\text { Observed, } \\
\text { with delay }\end{array}$ & $\begin{array}{l}\text { Continuous, } \\
\text { with a one- } \\
\text { quarter lag }\end{array}$ \\
\hline $\begin{array}{l}\text { Repatriate to the Treasury the proceeds of oil shipments } \\
\text { commercialized by private companies and SNPC on behalf of } \\
\text { the government within } 45 \text { days after the actual shipment date, } \\
\text { based on actual quantities, prices, and shipment dates. }\end{array}$ & Observed & Observed & Continuous \\
\hline
\end{tabular}


Table 7. Republic of Congo: Access and Phasing Under the 3-Year ECF Arrangement (2008-11) ${ }^{1}$

\begin{tabular}{|c|c|c|c|}
\hline \multirow[t]{2}{*}{ Timing } & \multicolumn{2}{|c|}{ Disbursement } & \multirow[t]{2}{*}{ Conditions } \\
\hline & $\begin{array}{l}\text { Amount } \\
\text { in SDRs }\end{array}$ & $\begin{array}{l}\text { Percent } \\
\text { of quota }\end{array}$ & \\
\hline $12 / 22 / 2008$ & $1,208,570$ & 1.43 & Approval of the arrangement \\
\hline $6 / 24 / 2009$ & $1,208,570$ & 1.43 & Review completed (July 2009) \\
\hline $12 / 4 / 2009$ & $1,208,570$ & 1.43 & Review completed (November 2009) \\
\hline $8 / 30 / 2010$ & $1,208,570$ & 1.43 & Review completed (August 2010) \\
\hline $1 / 11 / 2011$ & $1,208,570$ & 1.43 & Review completed (January 2011) \\
\hline $6 / 30 / 2011$ & $1,208,570$ & 1.43 & Completion of the fifth review (end-December 2010 test date) \\
\hline $7 / 30 / 2011$ & $1,208,580$ & 1.43 & Completion of the sixth (final) review (end-March 2011 test date) ${ }^{2}$ \\
\hline Total & $8,460,000$ & 10.00 & \\
\hline
\end{tabular}

${ }^{1}$ The Republic of Congo's quota is SDR 84.6 million.

${ }^{2}$ Given that the fifth and sixth reviews have been combined, the authorities have requested a rephasing in order to better align the disbursement with the Board date. 


\section{APPENDIX 1-SUPPLEMENTAL LETTER OF INTENT}

Brazzaville, July 14, 2011

The Minister of Finance, Budget

and Public Portfolio

To:

Ms. Christine Lagarde

Managing Director

International Monetary Fund

Washington, DC 20431

United States of America

Dear Managing Director:

Since reaching the HIPC Completion Point in January 2010, the Fund's Executive Board completed the third and fourth reviews of Congo's Extended Credit Facility (ECF) arrangement in August 2010 and January 2011, respectively. The Congolese government continues to implement a medium-term economic and financial program aimed at generating balanced growth, low and stable inflation, and fiscal and external sustainability. It is also determined to maintain the momentum of economic reform in order to accelerate growth and durably reduce poverty. In this regard, we believe the program objectives elaborated in our Memorandum of Economic and Financial Policies (MEFP) of December 2010 remain relevant and achievable.

The implementation of our Fund-supported program continues to be satisfactory and we observed all the continuous and quantitative performance criteria for end-December 2010 and end-March 2011. Notably, despite encountering current spending pressures in mid-2010, we were able to achieve the end-December program target. We attribute this strong performance to prudent budget management and the emerging benefits of public financial management and tax and customs administration reforms, as well as some tax policy changes. In 2011 we have stepped up tax reform, and are on track to surpass our ambitious tax collection target of 2 additional percentage points of non-oil GDP. In terms of financing, the higher net domestic financing of government is mostly explained by lower oil revenues and higher foreign deposits.

Performance in the structural area has also been satisfactory. Over the period under review we observed the two structural benchmarks aimed at strengthening oil governance, and despite falling short of meeting the structural benchmark percentage on procurement, mainly 
due to previously signed contracts and their regularization, we are pleased with our progress on implementing the new procurement code. After facing significant teething problems early on at the ministerial level, procurement procedures are now solidly in place and transparency has been increased through the posting of our annual procurement report online. I can now report that all ministries have approved procurement plans in place for 2011 and as of endMay 90 percent of contracts above $\$ 500,000$ have been subject to competitive bidding.

Building on our progress to date, we are committed to sustaining consolidation efforts through the end of the program and beyond, opening fiscal space for important development spending through lasting gains in non-oil revenue collection and restraint over non-essential current expenditure. As envisaged earlier, we project a basic primary non-oil deficit of CFAF 750 billion at end-December 2011 (34.4 percent of non-oil GDP). This includes a 30 percent increase in domestically financed capital expenditure aimed at addressing development needs. As a result, the fiscal adjustment over the course of the three-year ECF arrangement would be equivalent to a reduction of about 10 percent of non-oil GDP, as envisioned at the time of approval of the arrangement. Looking ahead, with infrastructure investment well underway, the 2012 budget will place increasing focus on improving social service provision in the areas of health and education, and on improving the quality of life of the Congolese people, including by bringing electricity and water to the main urban centers.

HIPC debt relief has led to a significant improvement in the government's external position and we will continue to maintain a prudent external borrowing policy. This includes both borrowing on concessional terms and enhancing our debt management capacity in order to preserve external stability.

We will support these policy objectives through determined efforts to improve tax policy, safeguard investment efficiency and strengthen governance and transparency, especially in the areas of oil wealth management and fiscal operations. In this regard, in 2011 we will take further measures to reform the tax system and step up implementation of our action plan to improve public financial management, including measures to strengthen our monitoring and accounting of fiscal operations. Building on reforms put in place in the context of the HIPC process, we will continue to work toward EITI compliance.

The Congolese government highly values technical assistance, and has taken the lead in providing financial support to AFRITAC in order for it to continue to perform its important role of capacity building in the region. It is with the help of AFRITAC that we are making progress in using a functional classification to enhance the monitoring of poverty-related spending. The Poverty Reduction Committee is studying the proposal made by AFRITAC to ensure that the recommendations are in line with the poverty profile. Once this validation is completed, we will closely monitor such spending to gauge our progress in addressing social challenges. 
As Chairman of the ministerial committee of the Central African Economic and Monetary Community (CEMAC) and the Board of Directors of the regional central bank (BEAC), we will strive to comply with our obligations under CEMAC including repatriation by the end of the year, address the weaknesses in BEAC and strengthen regional integration.

With the final test date under the program successfully behind us, it is with great pride that we request the completion of the fifth and sixth reviews and the disbursement of the sixth and seventh loans under the ECF arrangement. We also request that the seventh disbursement be re-phased to end-July in order to better align it with completion of the sixth and final review and subsequent expiration of the program.

However, while the program is coming to a close it is our firm intention and desire to remain closely engaged with the Fund, benefitting from its advice and technical assistance to enhance policy making and durably reduce poverty. In the future we will continue to consult closely with Fund staff providing all necessary information required for effective economic surveillance. The government intends to make the contents of this letter and those of the attached Technical Memorandum of Understanding (Attachment 1), as well as the staff report accompanying its request for completion of the fifth and sixth reviews of the program, available to the public and authorizes the Fund to arrange for them to be posted on the Fund's website, subsequent to Executive Board approval of its request.

Sincerely yours,

$/ \mathrm{s} /$

Gilbert Ondongo

Minister of Finance, Budget, and Public Portfolio

Attachment 
Table 1. Republic of Congo: Quantitative targets, 2010-11

(Billions of CFA francs; cumulative from January; unless otherwise indicated)

\begin{tabular}{|c|c|c|c|c|c|c|}
\hline & \multicolumn{2}{|c|}{ End-Jun. 2010} & \multicolumn{2}{|c|}{ End-Dec. 2010} & \multicolumn{2}{|c|}{ End-March 2011} \\
\hline & $\begin{array}{l}\text { Perf. } \\
\text { Criteria }\end{array}$ & Est & $\begin{array}{l}\text { Perf. } \\
\text { Criteria }\end{array}$ & Est. & $\begin{array}{l}\text { Perf. } \\
\text { Criteria }\end{array}$ & Prel. \\
\hline \multicolumn{7}{|l|}{ Quantitative targets } \\
\hline Basic Non-oil primary fiscal balance (floor) & -363 & -350 & -663 & -658 & -189 & -188 \\
\hline $\begin{array}{l}\text { New medium or long-term nonconcessional external debt } \\
\text { (including leasing) contracted or guaranteed by the government } \\
\text { (ceiling) }^{1,2,3}\end{array}$ & 0 & 0 & 0 & 0 & 0 & 0 \\
\hline $\begin{array}{l}\text { New external debt (including leasing) with an original maturity of } \\
\text { less than one year (ceiling) }{ }^{2}\end{array}$ & 0 & 0 & 0 & 0 & 0 & 0 \\
\hline $\begin{array}{l}\text { New oil-collateralized external debt contracted by or on behalf } \\
\text { of the central government (ceiling) }{ }^{2}\end{array}$ & 0 & 0 & 0 & 0 & 0 & 0 \\
\hline $\begin{array}{l}\text { New nonconcessional external debt with a maturity of more than } \\
1 \text { year contracted or guaranteed by SNPC (ceiling) }{ }^{2}\end{array}$ & 0 & 0 & 0 & 0 & 0 & 0 \\
\hline New external arrears on nonreschedulable debt (ceiling) ${ }^{2}$ & 0 & 0 & 0 & 0 & 0 & 0 \\
\hline New domestic arrears ${ }^{2}$ & 0 & 0 & 0 & 0 & 0 & 0 \\
\hline \multicolumn{7}{|l|}{ Memorandum items } \\
\hline Oil revenue & 1,060 & 910 & 1,949 & 1,758 & 595 & 544 \\
\hline Non-oil primary revenue & 211 & 227 & 465 & 463 & 137 & 137 \\
\hline $\begin{array}{l}\text { Net domestic financing of the government } \\
\text { (ceiling, indicative target) }\end{array}$ & -618 & -188 & -668 & -289 & -383 & -101 \\
\hline
\end{tabular}

\footnotetext{
${ }^{1}$ Excluding rescheduling arrangements and disbursements from the IMF; the minimum grant element is set to 35 percent.

${ }^{2}$ Continuous.

${ }^{3}$ The zero ceiling on nonconcessional external debt does not apply to forthcoming external loans from the European Investment Bank and the Central African States Development Bank, as specified in paragraph 11 of the Technical Memorandum of Understanding.
} 
Table 2. Republic of Congo: Structural Benchmarks under the ECF Arrangement, 2010-11

\begin{tabular}{|c|c|c|c|}
\hline Measures & $\begin{array}{l}\text { Status } \\
\text { End- Dec } \\
2010\end{array}$ & $\begin{array}{l}\text { Status } \\
\text { End-March } \\
2011\end{array}$ & Timing \\
\hline \multicolumn{4}{|l|}{$\begin{array}{l}\text { Public financial management and domestic revenue } \\
\text { mobilization } \\
\text { Justification: Strengthen investment quality }\end{array}$} \\
\hline $\begin{array}{l}\text { Support implementation of the new procurement code by } \\
\text { ensuring that } 80 \text { percent of public contracts having a total } \\
\text { value of over CFAF } 250 \text { million are open to competitive } \\
\text { bidding. }\end{array}$ & $\begin{array}{l}\text { Observed, } \\
\text { with delay }\end{array}$ & $\begin{array}{l}\text { Observed, } \\
\text { with delay }\end{array}$ & Continuous \\
\hline \multicolumn{4}{|l|}{$\begin{array}{l}\text { Governance and natural resource management } \\
\text { Justification: Continue strengthening of oil sector governance }\end{array}$} \\
\hline $\begin{array}{l}\text { Have an internationally reputable audit firm certify oil revenue } \\
\text { quarterly, using the same specifications as for the } 2003 \\
\text { certification and with no restrictions on access to the } \\
\text { information; and publish the certification reports on the } \\
\text { website of the ministry of economy, finance and budget } \\
\text { (www.mefb-cg.org). Also, the government will post on the } \\
\text { website not only the audit but for each report, a note } \\
\text { addressing comments by the auditors. }\end{array}$ & $\begin{array}{l}\text { Observed, } \\
\text { with delay }\end{array}$ & $\begin{array}{l}\text { Observed, } \\
\text { with delay }\end{array}$ & $\begin{array}{l}\text { Continuous, } \\
\text { with a one- } \\
\text { quarter lag }\end{array}$ \\
\hline $\begin{array}{l}\text { Repatriate to the Treasury the proceeds of oil shipments } \\
\text { commercialized by private companies and SNPC on behalf of } \\
\text { the government within } 45 \text { days after the actual shipment date, } \\
\text { based on actual quantities, prices, and shipment dates. }\end{array}$ & Observed & Observed & Continuous \\
\hline
\end{tabular}




\section{APPENDIX I-ATTACHMENT I \\ Technical Memorandum of Understanding}

Brazzaville, July 14, 2011

1. This technical memorandum of understanding (TMU) describes the definitions that are intended to clarify the measurement of the quantitative performance criteria and indicators in Table 1 of the Supplemental Letter of Intent (July 14, 2011) and the Memorandum of Economic and Financial Policies (MEFP, dated December 20, 2010) covering 2010-11. All quantitative performance criteria and indicators for 2011 will be evaluated in terms of cumulative flows from December 31, 2010. Also, the TMU specifies the periodicity and deadlines for transmission of data to the staff of the IMF for program monitoring purposes.

\section{DEFinitions AND Computation}

\section{A. Government}

2. Unless otherwise indicated, government is defined as the central government of the Republic of Congo and does not include local governments, the central bank, and any public entity with autonomous legal personality (i.e., wholly- or partially-owned state enterprises) not currently covered by the government's consolidated financial operations table (tableau des opérations financières de l'Etat-TOFE).

\section{B. Basic Primary Fiscal Balance}

3. The scope of the government's financial operations table (TOFE) includes the general budget and the special accounts of the Treasury (including the forestry and road funds) and the government debt management agency (Caisse Congolaise d'Amortissement, CCA).

4. The government's non-oil basic primary fiscal balance is defined as total non-oil revenue excluding grants and investment income (on the government's accounts in the central and commercial banks), minus total expenditure (including net lending), which is to exclude transfers to Hydro Congo, interest payments on debt, externally-financed capital expenditure, and capital expenditure financed by debt services savings under the enhanced Heavily Indebted Poor Countries (HIPC) Initiative. It is calculated from the budget execution outturn reported every month in the TOFE prepared by the ministry in charge of finance. 
5. The government's total revenue is reported in the TOFE on a cash basis. It includes all revenue collected by the Treasury (from tax and customs receipts, oil, services and forestry), whether they result from past, current, or future obligations. Receipts also include those recorded on a gross basis, in special accounts.

6. Oil revenue is defined as the government's net proceeds from the sale of oil, including the provision for diversified investments, royalties paid by oil companies, and the government's share of excess and profit oil. It excludes all forms of prepayment and pre-financing. The oil revenue projections take account of the 45 day lag between the date of shipment and the date of receipt of the sale proceeds by the Treasury.

7. Expenditures are recorded on a payment order basis. They include current expenditure, domestically-financed capital expenditure, foreign-financed capital expenditure, and net lending. Current expenditures include expenditures on wages, goods and services, common charges, interests on debt (domestic and external), transfers and subsidies, and other current expenditures. Subsidies to the state-owned oil refinery, CORAF, are estimated on the basis of the enterprises income statement.

8. Pro-poor spending is defined in the budget on the basis of the functional classification.

\section{Net domestic financing of the central government}

9. Net domestic financing (NDF) of the central government is defined as the change in the government's net position (claims minus deposits at the BEAC and commercial banks) in the banking system, which is elaborated in the table of Position Nette du Gouvernement table (PNG). NDF also includes the change in the stock of Treasury bills and bonds issued by the Congolese Treasury in CFA francs on the CEMAC regional market, when those securities are established but exclude the change in the outstanding of IMF credits at the BEAC (recours au dredits due FMI) . At end-December 2010, NDF was assessed at -1,269.29 billion CFAF. For each test date, any adjustment by the BEAC to the data reported by individual commercial banks, on account of their misclassification of government or for other reasons, will be reported to the Fund.

\section{Foreign Debt and External Arrears}

10. The definition of government used for the various external debt indicators includes government, as defined in paragraph 2, public institutions of an administrative nature (Etablissements Publics Administratifs), public institutions of a scientific and/or technical nature, public institutions of a professional nature, public institutions of an industrial and/or commercial nature (Entreprises Publiques d'Intérêt Commercial), and local governments, with the sole exception of the national oil company (SNPC) - see paragraph 14 below. It is defined on the basis of residency of the creditor. 
11. For the purposes of this memorandum, debt and concessional loans are defined as follows:

As specified in the guidelines adopted by the Executive Board of the IMF, ${ }^{1}$ debt will be understood to mean a current, i.e., not contingent, liability, created under a contractual arrangement through the provision of value in the form of assets (including currency) or services, and which requires the obligor to make one or more payments in the form of assets (including currency) or services, at some future point(s) in time; these payments will discharge the principal and/or interest liabilities incurred under the contract. Debts can take a number of forms, the primary ones being as follows: (i) loans, i.e., advances of money to the obligor by the lender made on the basis of an undertaking that the obligor will repay the funds in the future (including deposits, bonds, debentures, commercial loans and buyers' credits) and temporary exchanges of assets that are equivalent to fully collateralized loans under which the obligor is required to repay the funds, and usually pay interest, by repurchasing the collateral from the buyer in the future (such as repurchase agreements and official swap arrangements); (ii) suppliers' credits, i.e., contracts where the supplier permits the obligor to defer payments until some time after the date on which the goods are delivered or services are provided; and (iii) leases, i.e., arrangements under which property is provided which the lessee has the right to use for one or more specified period(s) of time that are usually shorter than the total expected service life of the property, while the lessor retains the title to the property. The debt is the present value (at the inception of the lease) of all lease payments expected to be made during the period of the agreement, excluding those payments that cover the operation, repair, or maintenance of the property. Under the definition of debt set out above, arrears, penalties, and judicially awarded damages arising from the failure to make payment under a contractual obligation that constitutes debt are debt. Failure to make payment on an obligation that is not considered debt under this definition (e.g., payment on delivery) will not give rise to debt.

Loan concessionality is assessed on the basis of the commercial interest reference rates (CIRRs) established by the OECD. A loan is said to be on concessional terms if, on the date of conclusion of the contract, the ratio of the net present value of the loan, calculated on the basis of the reference interest rates, to its nominal value is less than 35 percent (i.e., a grant element of at least 35 percent). ${ }^{2}$ For debts with a maturity exceeding 15 years, the ten-year reference interest rate

\footnotetext{
${ }^{1}$ See Executive Board Decision No. 6230-(79/140) as amended by Decisions Nos. 11096-(95/100), 12274(00/85) and 14416- (09/91), August 31, 2009, effective December 1, 2009.

${ }^{2}$ See "Staff Guidance Note on Debt Limits in Fund-Supported Programs", December 18, 2009.
} 
published by the OECD is used to calculate the grant element. For shorter maturities, the six-month market reference rate is used.

The concessionality requirement applies not only to the central government, but also covers debt incurred by public enterprises. The sole exception to this concessionality requirement is the projected external loans to the Port Authority of Pointe Noire to support its rehabilitation from the European Investment Bank in an amount up to euro 29 million, and from the Central African States Development Bank in an amount up to euro 9.1 million.

12. The quantitative indicative target with respect to external debt apply not only to debt as defined in the above-mentioned guidelines, but also to commitments incurred or guaranteed for which no value has yet been received or on which only partial drawings have been made. However, this does not apply to financing from the IMF or to Treasury bills and bonds issued by the Congolese Treasury in CFA francs on the CEMAC regional market.

13. For external debt with an initial maturity of less than one year (a continuous quantitative performance criterion), normal short-term import and export credit are excluded from the scope of the indicator, including the prepayments.

14. The ceiling on any new nonconcessional external debt with a maturity of more than one year contracted or guaranteed by the SNPC, with or without government guarantee, will be observed continuously. The SNPC may borrow only to finance investments related to its core activities (research, exploration, production, refining and distribution of oil, construction of a Brazzaville headquarters, creation and strengthening of its database, etc.). In addition, these investments must be included in the SNPC's investment budget approved by its board of directors. The ceiling on debt does not apply to changes in loan accounts with oilfield partners or to loans with maturities of less than one year.

The accumulation by the government of external payment arrears is the difference between (i) the gross amount of external debt service payments due (principal and interest, including penalty and/or late interest, as appropriate) and (ii) the amount actually paid during the period under consideration. Under the program, the government commits itself to not accumulate external payment arrears on non-reschedulable debt (that is, debt to Paris Club creditors contracted after the cutoff date and debt to multilateral creditors). Nonaccumulation of external payment arrears is an indicator to be continuously observed.

\section{E. Oil-Collateralized External Debt and Oil Prepayments}

15. Oil-collateralized external debt is external debt which is contracted by giving an interest in oil. Pre-financing is defined as an oil-collateralized loan which is repaid by the sale of the oil in a different calendar year. New pre-financing by or on behalf of the 
government is strictly prohibited under the program. The refinancing and/or deferral of the existing stock and/or due dates are permitted but should not give rise to an augmentation of the existing stock of oil-collateralized debt.

16. A prepayment is defined as an advance payment by the purchaser of oil on a specific oil shipment. Prepayment-related operations must be repaid within 6 months, but in any case within the calendar year in which they were contracted.

\section{F. Payment Arrears and Domestic Debt}

17. Domestic payment arrears of the government are equivalent to the difference over the period under review between the amount of payments authorized and the actual payments made (within 90 days).

\section{INFORMATION FOR Program MONITORING}

18. The government will submit the following information to the staff of the IMF through its Resident Representative, and within the time period specified below.

\section{A. Oil Sector}

19. Regarding the oil sector, the government will submit the following information to IMF staff within four weeks after the end of the month:

the monthly data on oil production by oil field, production costs, volume exported, export prices, and the operations of the national oil company (SNPC);

the breakdown concerning the share of crude oil that accrues to the government, by oil field, distinguishing the type of resource to which this share relates (royalties, profit oil, etc.);

any change in the tax parameters;

a breakdown of oil prices;

a monthly detailed list of shipments commercialized by SNPC on behalf of the government, including information on the type of product, the date of loading, the recipient, the number of barrels and the selling price (in US dollars and CFAF) as well as the date of receipt of the sale proceeds by the Treasury; and

actual and projected quarterly data to determine the required subsidies in the fuel sector, including prices, quantities, and costs. 


\section{B. Government Finance}

20. Regarding government finance, the government will submit the following information to IMF staff:

A table on government fiscal operations (TOFE) and its annexes. The annexed tables include (i) the breakdown of oil revenue in value terms with the corresponding notes on computation, (ii) excess oil trends and any bonus payments, (iii) the breakdown of tax and non-tax revenue, and central government expenditure, particularly transfers and common charges; and (iv) a report on the amounts of and rationales for emergency payment and cash advance procedures. The provisional TOFE and its annexes will be reported monthly within four weeks from the end of the month, whereas the final TOFE and its annexes will be reported within six weeks from the end of each month.

Monthly data on the prices and taxation of petroleum products. These data will include: (i) the price structure in effect during the month; (ii) the details of computation of the price structure, (f.o.b. Mediterranean price) at retail prices, including the border impact prices, taxes, transit costs, economic adjustments, exrefinery prices (for CORAF and imports), entry distribution prices, margins and fees, transport costs and losses, financing expenses, and insurance; (iii) amounts released for sale; and (iv) a breakdown of the tax revenue from oil productscustoms duties and value-added tax - and direct/indirect subsidies incurred by the budget. These data will be reported within four weeks from the end of the month.

The Treasury balance to monitor expenditures. It will include the amount of commitments, payment orders, and payments, for both current and capital expenditure. It will be produced on a quarterly basis, and submitted to Fund staff no later than four weeks after the end of each quarter.

Data on implementation of the public investment program, including the breakdown relating to financing sources. If the data on the execution of investments financed with foreign grants and loans are not available on schedule, a linear estimate of execution in comparison with annual forecasts will be used. These data will be reported on a quarterly basis within four weeks from the end of the quarter.

Complete monthly data on domestic financing of the budget (net bank credit to, and net non-bank credit to the government). These data will be reported monthly within four weeks from the end of the month.

The table used to monitor the expenditure process will list the amount of commitments, payment orders, and payments, for both operating and capital 
expenditures. It will be produced on a quarterly basis, and submitted to Fund staff no later than four weeks after the end of the quarter.

A quarterly table for monitoring poverty reduction expenditures, based on the propoor sectors defined in the poverty reduction strategy paper-basic health care and education; infrastructure and rural integration; water and electricity; disarmament, demobilization and reintegration; social protection, and agriculture). The quarterly tables will be submitted within four weeks of the end of the quarter.

A monthly table of prepayments, which will also indicate the nature of the expenditures (current transfers, investment, etc.) and the justification for the need to use the prepayment option.

\section{Monetary Sector}

21. The government will submit on a monthly basis, within four weeks of the end of the month, the following preliminary information:

$>\quad$ the consolidated balance sheet of the monetary institutions, the central bank survey, and the commercial banks survey;

$>\quad$ the integrated monetary survey;

$>\quad$ the table of lending and deposit rates; and

the usual banking supervision indicators for banks and non-bank financial institutions, where necessary.

22. The final data for the integrated monetary survey will be transmitted within six weeks of the end of the month.

\section{Balance of Payments}

23. The government will submit the following information to IMF staff:

any revised balance of payments data (including services, private transfers, official transfers, and transactions for the capital and financial account) as soon as the data are revised; and

foreign trade statistics (volume and price) prepared by the national statistics agency within three months of the end of the reporting month. 


\section{E. Debt}

24. The government will submit the following to the staff of the IMF within four weeks of the end of the month:

$>\quad$ data on the stock, accumulation, and payment of domestic arrears;

$>\quad$ data on the stock, accumulation, and payment of external payment arrears;

a breakdown of estimated domestic and external public debt service, service due, and actual payments, including breakdowns of principal and interest and by creditor;

the list and amounts of new external debt incurred or guaranteed by the government, including detailed information on the terms and conditions (currency, interest rate, grace period, and maturity) stated in the original agreement; and

actual disbursements of foreign financial assistance (project and non-project), including new borrowing and any external debt relief granted by foreign creditors (CCA tables).

\section{F. Real Sector}

25. The government will submit the following to the staff of the IMF:

monthly itemized consumer price indices, within four weeks of the end of the month;

$>\quad$ any revision of the national accounts; and

any other indicators and statistical data used to track overall economic developments, including information on activity in the forestry sector and woodprocessing industry, as well as the short-term economic bulletins prepared monthly.

\section{G. Structural Reforms and Other Data}

26. The government will submit the following information to the IMF staff:

a monthly detailed table concerning the implementation of structural measures under the program;

any study or official report on the economy of the Republic of Congo, within two weeks of its publication; and 
any decision, order, law, decree, ordinance, or circular having economic or financial implications for the program, within two weeks from the time it is published, or, at the latest, from its entry into force. 


\title{
INTERNATIONAL DEVELOPMENT ASSOCIATION AND INTERNATIONAL MONETARY FUND
}

\author{
REPUBLIC OF CONGO \\ Joint IMF/World Bank Debt Sustainability Analysis
}

Prepared by the Staffs of the International Monetary Fund and the International Development Association ${ }^{1}$

Approved by Domenico Fanizza and Dhaneshwar Ghura (IMF)

Sudarshan Gooptu and Marcelo Giugale (IDA)

July 15, 2011

Based on the Debt Sustainability Framework (DSF) for Low Income Countries (LIC), the rating for the Republic of Congo shifts to low from moderate risk of debt distress. Under the baseline, none of the indicative thresholds are breached. There is one brief and small breach of the PV of debt-to-GDP threshold when stress tested, which could however be financed from accumulated deposits rather than external debt. Considering accumulated liquid assets, external debt is resilient to shocks, but public finances remain vulnerable to oil price volatility and the maturing of wells over the longer run. Consequently, consolidation is still required to ensure fiscal sustainability and strengthen external stability-fiscal discipline, pro-growth structural reforms and efficient public investment are key to keeping the overall balance in surplus while raising growth. Continued improvements in debt management and development of an oil wealth management strategy would further reduce risks.

\section{Background}

1. Congo's net external debt is low. Congo reached the HIPC Completion Point in January 2010, and in March 2010 the Paris club granted 100 percent relief. At end-2010, Congo's net external debt is estimated at $3 \frac{1}{2}$ percent of GDP, with gross official foreign assets and gross official debt amounting to 44 and $471 \frac{1}{2}$ percent of GDP, respectively. Public and publicly guaranteed debt (PPG) comprises roughly half of external debt, with the remainder owed mostly by the national oil company. ${ }^{2}$ In 2010 , domestic government debt

\footnotetext{
${ }^{1}$ In collaboration with the Congolese country authorities.

${ }^{2}$ Given data limitations, PPG covers only debt of the central government. However, wage and pension arrears of restructured state owned enterprises have been assumed by government and are included in domestic debt.
} 
amounted to 2.6 percent of GDP and constituted wage and pension arrears of restructured state owned enterprises which have been assumed by government and are subject to a repayment plan.

\section{Macroeconomic assumptions}

2. The baseline macroeconomic framework for the debt sustainability analysis reflects current policies and macroeconomic prospects. The key macroeconomic assumptions are summarized in Box 1. The outlook is favorable, with the increasing stock of basic infrastructure supporting an acceleration of non-oil growth. Growth in 2011-13 averages 7.5 percent as a result of robust activity in the oil, telecom, construction, timber and transport sectors. Over the medium term, non-oil growth gains further momentum as completion of public investment projects eases transport and supply bottlenecks, while structural reforms to enhance the business climate begin to take hold. The successful completion of structural reforms and broader private sector participation in economic activity sustain growth in the long run. The external position continues to strengthen as Congo's sizable oil wealth and high petroleum prices limit the need for external borrowing and allow for a buildup of government assets. As Congo begins to approach emerging market status (expected in 2025), total external debt is projected to start to tick up as the private sector begins to borrow from international markets.

\section{External Debt Sustainability Analysis}

3. Under the baseline scenario, gross external debt averages 20 percent of GDP and net external debt is negative over the entire projection period. The present value of gross PPG external debt remains well below all indicative thresholds (Figure 1), and the large buildup of government deposits (i.e., asset accumulation) resulting from sustained fiscal surpluses implies falling external vulnerability. ${ }^{3}$

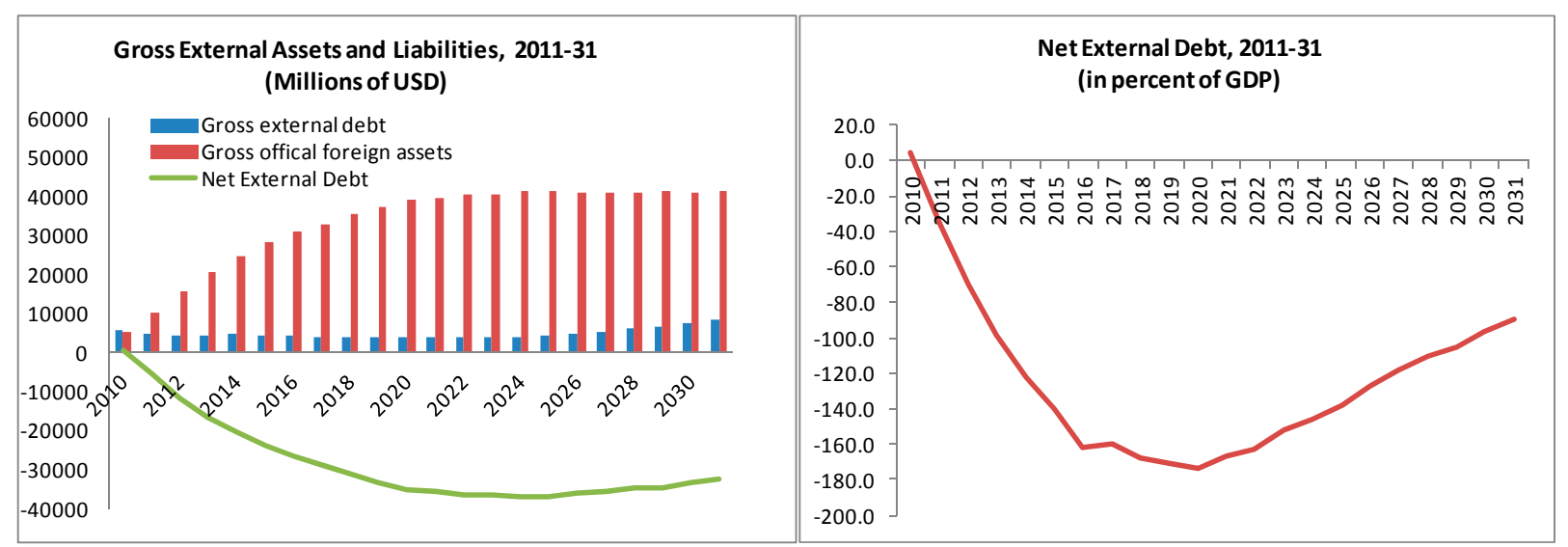

\footnotetext{
${ }^{3}$ The 3-year moving average of the CPIA indicates that the country is a weak performer.
} 


\section{Box 1. Republic of Congo: Baseline Macroeconomic Assumptions}

Real GDP growth: Over 2011-31 oil production declines and growth is increasingly led by non-oil activity. Over the medium term, public investment begins to narrow infrastructure gaps and non-oil growth is assumed to rise significantly (average 8.6 percent) driven by robust activity in the telecoms, construction, timber and transportation sectors. Over the longer term, assumed structural changes and reforms support growth through an enhanced business climate, and increasing infrastructure (rail, roads and ports) supports new industries such as mining and light manufacturing, including agroindustry.

Oil production and prices: Oil production is expected to peak in 2011 at about 126 million barrels per year and decline thereafter to 21 million barrels per year in 2031. Current WEO oil prices average about $\$ 100$ per barrel over the medium term, and are assumed to remain constant in real dollar terms thereafter.

Inflation: Inflation would subside over the medium term (4 percent average) as infrastructure bottlenecks ease, stabilizing at around 3 percent, in line with the Central African Economic and Monetary Community (CEMAC) convergence criterion ( 3 percent).

Balance of payments: The current account would remain in surplus over $2011-19$ ( $8 \frac{1}{2}$ percent average) before swinging into deficit starting in 2020 (-83/4 percent average) as oil production dwindles. In later years, the trade deficit would be partially offset by interest on foreign assets and lower profit remittances by oil companies. FDI in the non-oil sector is projected to grow by around 12 percent per year as the investment environment improves and new sectors such as mining are opened to foreign investors; the share of the non-oil sector in FDI is projected to increase to around 90 percent (13 percent in 2010). Gross official foreign assets would stabilize at around $\$ 40$ billion and decline over time as a percent of GDP.

Government balance: The government is expected to continue consolidation efforts aimed at long-term fiscal sustainability while at the same time investing to meet country's significant development needs. The overall fiscal balance is projected to decline with oil production but remain in surplus over the longer term. As a result, government deposits would gradually decline to around 100 percent of GDP in 2031. Under such a scenario, there is no borrowing need. However, the authorities are assumed to fully draw the estimated $\$ 1.6$ billion Chinese line of credit associated with nine infrastructure projects, as well as lending in the pipeline from international financial institutions.

External assistance: As the country progresses toward an emerging economy, grants (mainly technical assistance) and loan concessionality are assumed to diminish. From 2019, Congo is expected to obtain limited amounts of non-concessional loans from the IBRD.

Domestic borrowing: Domestic arrears are fully repaid by 2014, and no new domestic borrowing is assumed over the projection period given Congo's abundant oil wealth. 
4. Given the public sector asset accumulation under the baseline, results for the alternative scenarios and bound tests must be interpreted with caution. ${ }^{4}$ First, the alternative scenarios assume that any gap is filled by external borrowing rather than a smaller accumulation of assets. Second, 10-year historical averages are likely poor proxies of future outcomes for countries experiencing an oil boom and significant growth-enhancing structural change.

5. Despite these caveats, debt dynamics for PPG external debt remain robust under both the alternative scenarios and bound tests. While holding key variables at their historical level (Table 2, scenario A.1) leads to a slight breach of the indicative threshold of the present value of debt-to-GDP ratio in 2014-15, the gross debt stock would rise by only 15 percentage points (average) over 2011-15, and leave negative net debt in excess of 100 percent of GDP. The most extreme scenario is represented by the shock to exports. Under such circumstances, the threshold is breached for 6 years, peaking at 38 percent. The PV of

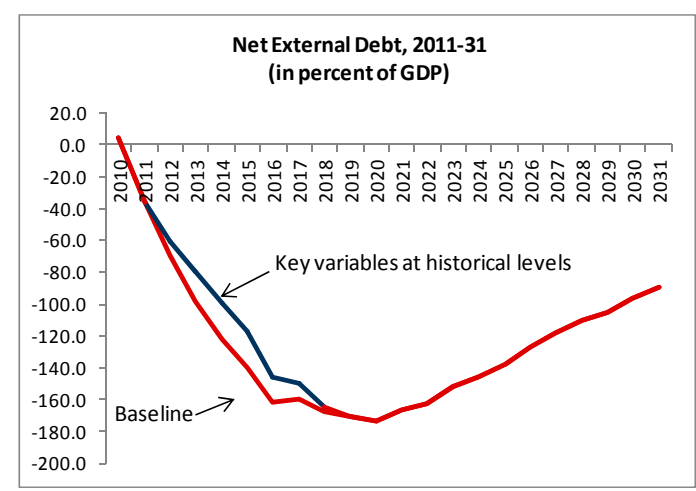
debt-to-exports and debt-to-revenue ratios, as well as the liquidity indicators remain well below relevant thresholds under both the alternative scenarios and standard bound tests (Figure 1).

6. While the DSA points to low risk of external debt distress, the prolonged fiscal consolidation assumed under the baseline is required for fiscal sustainability and external stability. Declining oil production and uncertainty regarding oil prices call for further strengthening of policy buffers. Over the medium term, the public sector should continue to borrow only on concessional terms, preferably for high return projects containing a large technical assistance component. As market access increases the authorities should maintain a cautious borrowing policy.

\section{Public Sector Debt Sustainability}

7. Under the baseline scenario public and publicly guaranteed debt remains low and net public external debt is negative. Public sector debt mirrors the external debt path, reflecting the low level of domestic debt and the lack of market access of the private sector.

\footnotetext{
${ }^{4}$ The LIC DSA template is ill equipped to analyze oil-rich countries with negative debt which experience asset accumulation over the projection period. Alternatively, the template could be run using net debt.
} 


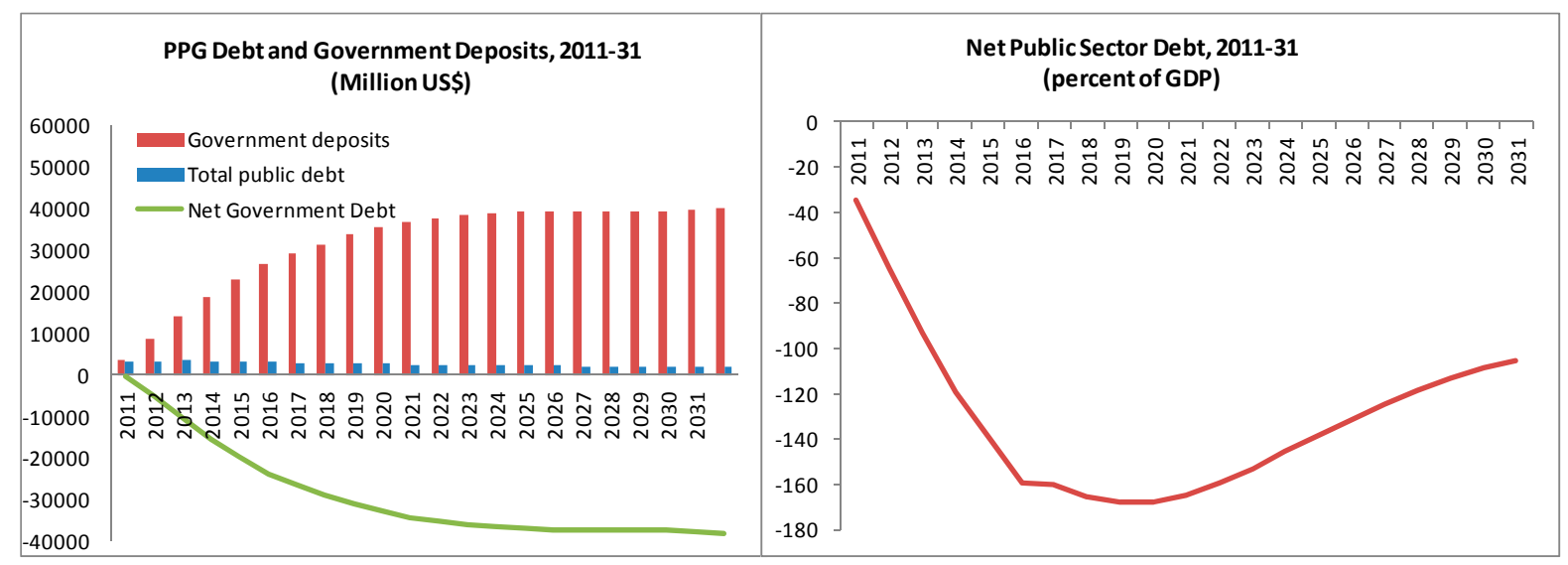

8. Debt dynamics are most sensitive to a lower primary surplus resulting from lower oil revenues or the overspending of oil wealth. Under the baseline, the primary balance registers a surplus of more than 28 percent of GDP (average) over the medium term, declining rapidly thereafter as oil production wanes, but remaining large at over 4 percent of GDP at the end of the projection period. Under the alternative scenario, with real GDP growth and the primary balance at historical averages (Table 4a, scenario A.1), debt dynamics worsen, with the largest impact coming from the lower (but still positive) primary balance while liquid government deposits keep accumulating. Thus, this shock appears irrelevant given the authorities' policy of financing development expenditure mostly with own resources - in the event of lower oil revenues the authorities would most likely accumulate fewer government deposits and/or adjust investment spending to available resource wealth. The most extreme shock is represented by the combination shock, which is a variation of the historical alternative scenario, only this time it is temporary rather than permanent. The same aforementioned analytical caveats also apply in this case.

\section{The vulnerability of Congo's public finances to oil price volatility puts a} premium on maintaining fiscal discipline. In addition to prudent spending, good debt management and cautious borrowing, efforts should focus on increasing non-oil revenueincluding through structural reforms to foster non-oil activity and tax policy-to safeguard long-term debt sustainability.

10. The authorities agreed with the assessment. The external position has improved significantly as a result of rising oil production, favorable oil prices and debt relief under the HIPC initiative. Looking ahead, the strong external environment together with their commitment to maintaining prudent debt policies - including financing public investment mostly through own resources - will allow them to accumulate net assets, thereby further reducing vulnerability. They recognize the vulnerability of the fiscal and external accounts to volatile oil prices, and are (a) taking steps to increase economic diversification through implementing an action plan to improve the business climate and (b) undertaking a tax reform to strengthen non-oil revenue collection. 


\section{Conclusion}

\section{Congo's external and public debt burden was significantly reduced after} reaching the HIPC completion point in January 2010 and the country is at a low risk of debt distress. At current oil price and public expenditure forecasts, Congo will not have a borrowing requirement over the medium to longer term. External debt is resilient to most shocks, but public finances remain vulnerable to oil price volatility. However, in the event of sharply lower oil prices, the government is unlikely to resort to extensive external borrowing, opting to accumulate fewer government deposits or adjust development expenditure to available oil resources.

\section{Despite the relatively positive external debt outlook, policy challenges remain.}

Historically, oil revenues have come in lower than staff estimates, and the renegotiation of oil contracts could lower the government's take of oil profits. Consolidation is still required to ensure fiscal sustainability and strengthen external stability - fiscal discipline, pro-growth structural reforms and efficient public investment are key to keeping the overall balance in surplus while raising growth. Continued improvements in debt management and development of an oil wealth management strategy would further reduce risks of debt distress. If an external financing need arises, the authorities should maintain a prudent external debt policy, borrowing only for high return projects and on concessional terms whenever possible. 
Figure 1. Republic of Congo: Indicators of Public and Publicly Guaranteed External Debt under Alternatives Scenarios, 2011-31 1/ 2/

a. Debt Accumulation
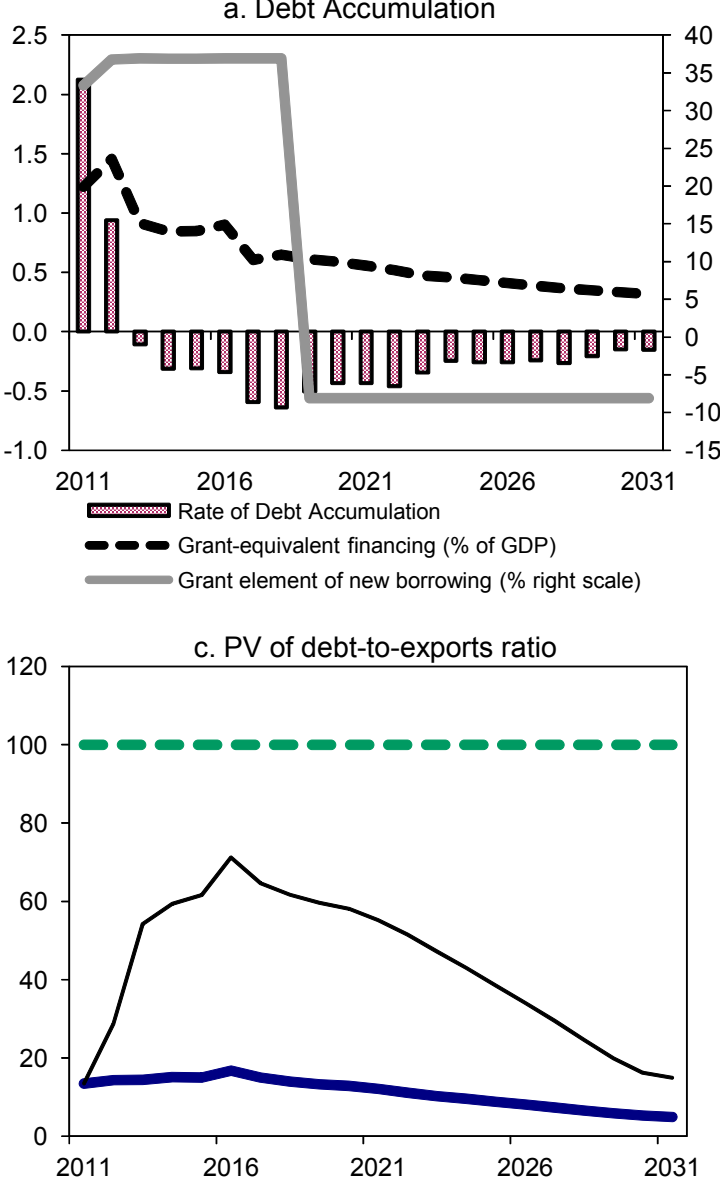

e. Debt service-to-exports ratio

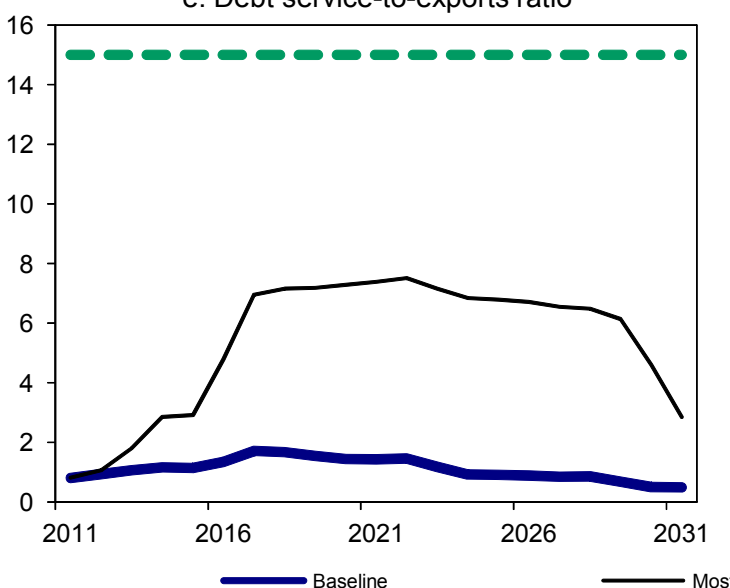

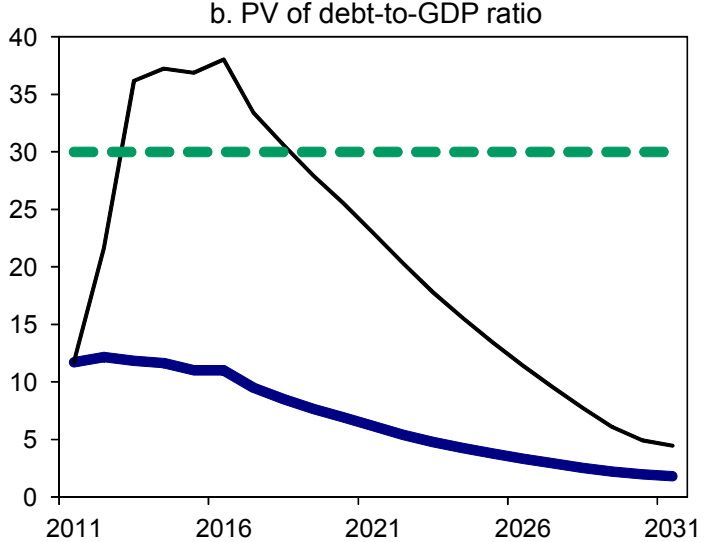

d. PV of debt-to-revenue ratio

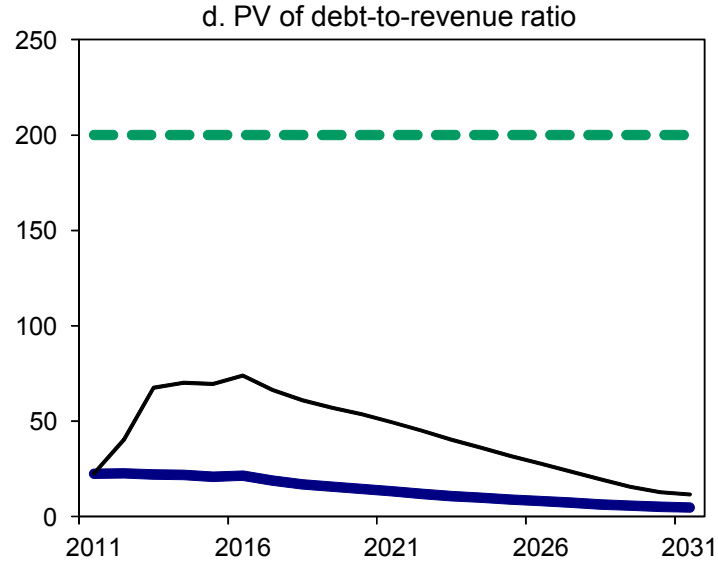

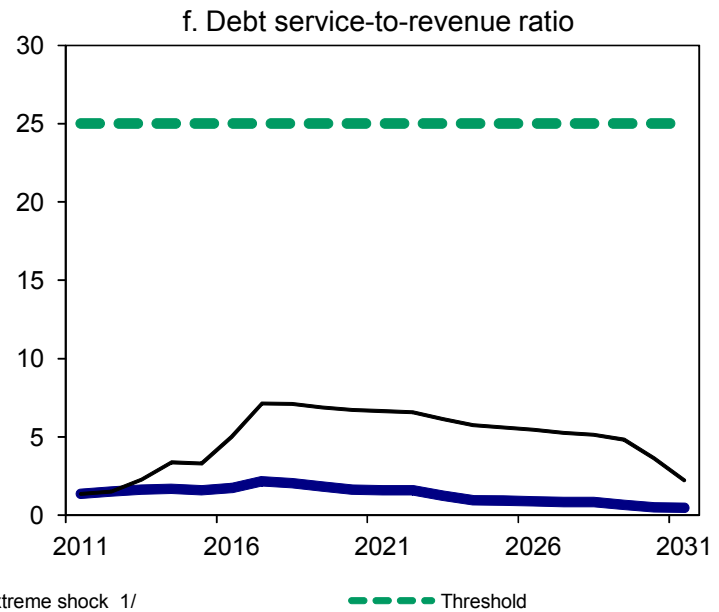

Sources: Country authorities; and staff estimates and projections.

$1 /$ The most extreme stress test is the test that yields the highest ratio in 2021. In all cases the most extreme shock is the export shock.

2/ The fixed primary balance scenario is deleted as it shows a negative net debt. 
Figure 2. Republic of Congo: Indicators of Public Debt Under Alternative Scenarios, 2011-31 1/ 2/
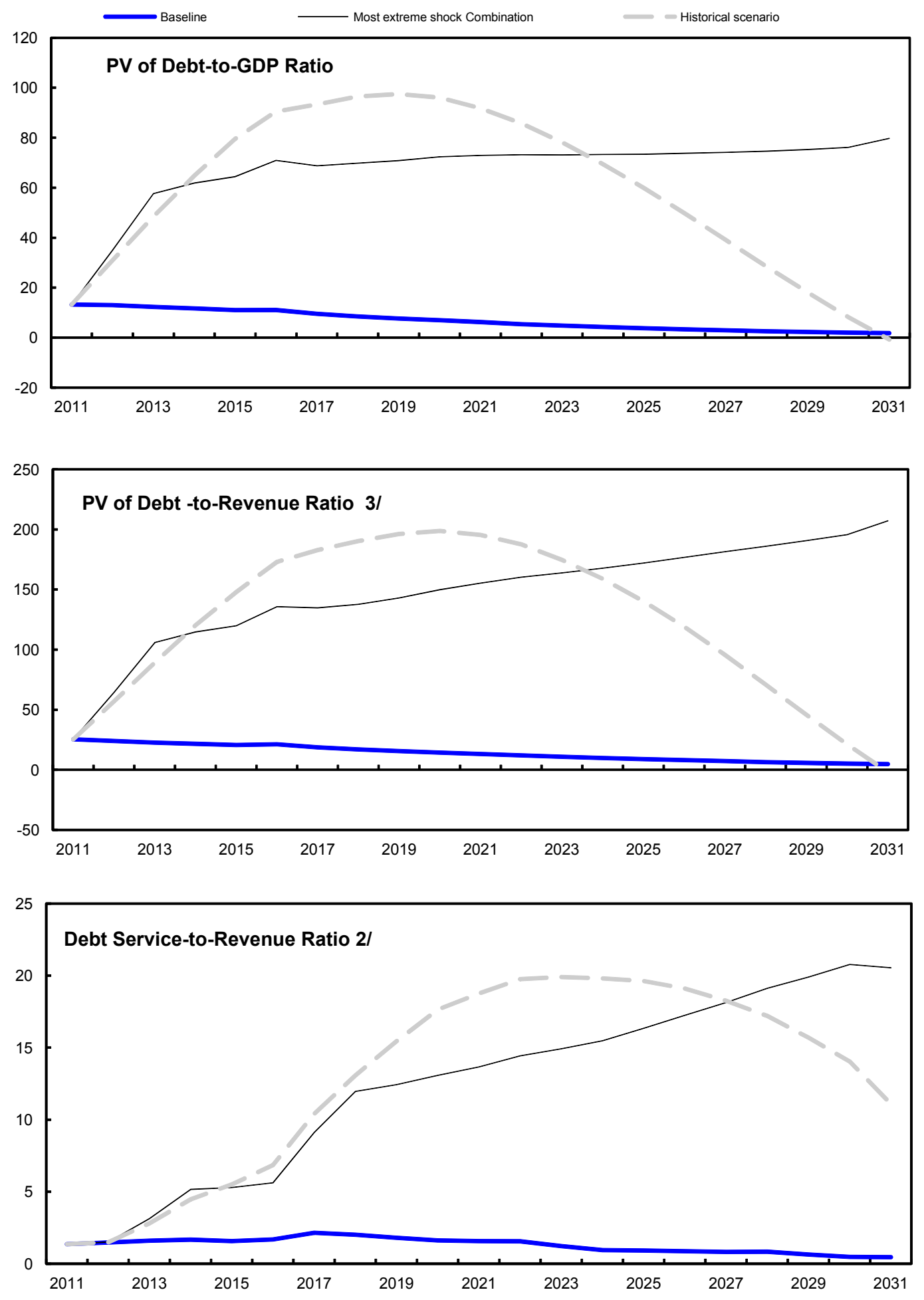

Sources: Country authorities; and staff estimates and projections.

$1 /$ The most extreme stress test is the test that yields the highest ratio in 2021. 2/The historical scenario has been suppressed, as it yields negative values. 3 / Revenues are defined inclusive of grants. 
Table 1. External Debt Sustainability Framework, Baseline Scenario, 2008-31 1/ (In percent of GDP, unless otherwise indicated)

\begin{tabular}{|c|c|c|c|c|c|c|c|c|c|c|c|c|c|c|c|}
\hline & \multicolumn{3}{|c|}{ Actual } & \multirow{2}{*}{\multicolumn{2}{|c|}{$\begin{array}{l}\text { Historical Standard } \\
\text { Average 6/ Deviation 6/ }\end{array}$}} & \multicolumn{6}{|c|}{ Projections } & \multirow[b]{2}{*}{$\begin{array}{l}2011-16 \\
\text { Average }\end{array}$} & \multirow[b]{2}{*}{2021} & \multirow[b]{2}{*}{2031} & \multirow[b]{2}{*}{$\begin{array}{l}2017-31 \\
\text { Average }\end{array}$} \\
\hline & 2008 & 2009 & 2010 & & & 2011 & 2012 & 2013 & 2014 & 2015 & 2016 & & & & \\
\hline External debt (nominal) 1/ & 89.2 & 84.5 & 47.7 & & & 30.3 & 25.2 & 25.6 & 27.7 & 26.6 & 26.4 & & 17.8 & 22.0 & \\
\hline o/w public and publicly guaranteed (PPG) & 59.7 & 50.2 & 22.7 & & & 19.6 & 20.2 & 19.5 & 19.2 & 18.2 & 18.2 & & 9.9 & 2.3 & \\
\hline Change in external debt & -43.8 & -4.7 & -36.8 & & & -17.4 & -5.1 & 0.4 & 2.1 & -1.1 & -0.3 & & $\begin{array}{l}-0.5 \\
-0.5\end{array}$ & 2.0 & \\
\hline Identified net debt-creating flows & -61.5 & 9.6 & -38.3 & & & -28.0 & -30.6 & -31.8 & -27.3 & -24.9 & -17.1 & & -7.7 & -1.4 & \\
\hline Non-interest current account deficit & -3.7 & 7.8 & -6.1 & -4.6 & 5.4 & -10.6 & -14.7 & -16.5 & -13.1 & -10.7 & -5.0 & & 1.8 & 6.4 & 3.6 \\
\hline Deficit in balance of goods and services & -27.0 & -19.7 & -33.8 & & & -36.2 & -37.0 & -36.0 & -28.5 & -23.3 & -12.4 & & 0.6 & 8.4 & \\
\hline Exports & 73.9 & 70.7 & 84.5 & & & 87.0 & 85.3 & 82.2 & 77.1 & 73.7 & 65.7 & & 51.2 & 36.8 & \\
\hline $\begin{array}{l}\text { Epporis } \\
\text { Imports }\end{array}$ & 47.0 & 51.0 & $\begin{array}{l}84.5 \\
50.7\end{array}$ & & & 50.8 & 48.3 & 46.2 & 48.6 & 50.5 & $\begin{array}{l}63.1 \\
53.3\end{array}$ & & 51.8 & $\begin{array}{l}35.1 \\
45.1\end{array}$ & \\
\hline Net current transfers (negative $=$ inflow) & $\begin{array}{l}47.0 \\
-0.1\end{array}$ & 0.4 & 0.6 & 0.5 & 0.2 & $\begin{array}{r}0.3 \\
0.3\end{array}$ & $\begin{array}{r}46.3 \\
-0.2\end{array}$ & 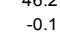 & $\begin{array}{c}40.6 \\
-0.1\end{array}$ & 0.0 & $\begin{array}{r}53.0 \\
0.0\end{array}$ & & $\begin{array}{r}3.0 \\
0.4\end{array}$ & $\begin{array}{l}3.1 \\
0.7\end{array}$ & 0.5 \\
\hline 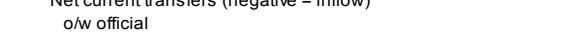 & $\begin{array}{l}-0.1 \\
-0.6\end{array}$ & $\begin{array}{r}0.4 \\
-0.2\end{array}$ & 0.0 & 0.5 & 0.2 & $\begin{array}{r}0.3 \\
-0.2\end{array}$ & $\begin{array}{l}-0.2 \\
-0.7\end{array}$ & $\begin{array}{l}-0.1 \\
-0.7\end{array}$ & $\begin{array}{l}-0.1 \\
-0.7\end{array}$ & -0.7 & -0.8 & & $\begin{array}{c}.4 .5 \\
-0.5\end{array}$ & $\begin{array}{c}.1 \\
-0.3\end{array}$ & 0.5 \\
\hline Other current account flows (negative = net inflow) & 23.3 & 27.1 & 27.2 & & & 25.3 & 22.5 & 19.6 & 15.5 & 12.6 & 7.4 & & 0.7 & -2.7 & \\
\hline Net FDI (negative = inflow) & -21.1 & -20.1 & -16.2 & -14.1 & 8.4 & -15.3 & -14.6 & -14.2 & -13.3 & -12.7 & -11.5 & & -8.8 & -7.5 & -8.5 \\
\hline Endogenous debt dynamics 21 & & 21.9 & & & & -2.1 & -1.2 & -1.1 & -1.0 & -1.4 & -0.6 & & -0.7 & -0.3 & \\
\hline 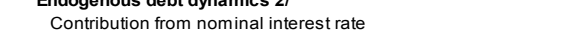 & $\begin{array}{r}-30.1 \\
2.5\end{array}$ & $\begin{array}{r}21.9 \\
1.1\end{array}$ & $\begin{array}{r}-16.0 \\
0.4\end{array}$ & & & 0.2 & 0.3 & 0.5 & 0.4 & 0.4 & 0.4 & & 0.3 & 0.3 & \\
\hline Contribution from real GDF & -5.2 & -8.2 & -6.0 & & & -2.3 & $\begin{array}{r}0.5 \\
-1.5\end{array}$ & -1.6 & -1.4 & -1.9 & -1.0 & & -1.0 & -0.6 & \\
\hline Contribution from price and exchange rate changes & -33.9 & 29.0 & -10.4 & & & & & & & & & & & & \\
\hline Residual (3-4) 3l & 17.7 & -14.3 & 1.6 & & & 10.6 & 25.4 & 32.2 & 29.4 & 23.8 & 16.8 & & 7.2 & 3.4 & \\
\hline o/w exceptional financing & -10.1 & -1.7 & -24.3 & & & 0.0 & 0.0 & 0.0 & 0.0 & 0.0 & 0.0 & & 0.0 & 0.0 & \\
\hline PV of external debt $4 /$ & 29.5 & 34.2 & 38.3 & & & 22.5 & 17.2 & 17.9 & 20.2 & 19.5 & 19.2 & & 14.1 & 21.5 & \\
\hline In percent of exports & 39.9 & 48.4 & 45.3 & & & 25.8 & 20.2 & 21.7 & 26.1 & 26.4 & 29.2 & & 27.5 & 58.4 & \\
\hline PV of PPG external debt & 29.5 & 34.2 & 13.3 & & & 11.7 & 12.2 & 11.8 & 11.6 & 11.0 & 11.0 & & 6.2 & 1.8 & \\
\hline In percent of exports & 39.9 & 48.4 & 15.7 & & & 13.5 & 14.3 & 14.4 & 15.1 & 15.0 & 16.8 & & 12.1 & 4.9 & \\
\hline In percent of government revenues & 63.8 & 99.0 & 35.1 & & & 22.5 & 22.7 & 22.1 & 21.9 & 20.8 & 21.5 & & 13.3 & 4.7 & \\
\hline Debt service-to-exports ratio (in percent) & 10.0 & 5.2 & 2.0 & & & 2.1 & 1.8 & 2.5 & 3.5 & 3.7 & 4.2 & & 4.2 & 5.0 & \\
\hline PPG debt service-to-exports ratio (in percent) & 5.2 & 3.7 & 1.3 & & & 0.8 & 0.9 & 1.1 & 1.2 & 1.1 & 1.3 & & 1.4 & 0.5 & \\
\hline PPG debt service-to-revenue ratio (in percent) & 8.3 & 9.0 & 2.9 & & & 1.4 & 1.5 & 1.6 & 1.7 & 1.6 & 1.7 & & 1.6 & 0.5 & \\
\hline Total gross financing need (Billions of U.S. dollars) & -2.1 & -0.8 & -2.5 & & & -3.8 & -4.5 & -4.8 & -3.9 & -3.5 & -2.3 & & -1.0 & 0.2 & \\
\hline Non-interest current account deficit that stabilizes debt ratio & 40.0 & 12.5 & 30.7 & & & 6.8 & -9.6 & -16.9 & -15.2 & -9.6 & -4.8 & & 2.3 & 4.4 & \\
\hline \multicolumn{16}{|l|}{ Key macroeconomic assumptions } \\
\hline Real GDP growth (in percent) & 5.6 & 7.5 & 8.8 & 4.7 & 3.2 & 6.3 & 5.3 & 6.4 & 5.4 & 6.9 & 3.7 & 5.7 & 5.9 & 2.8 & 5.0 \\
\hline GDP deflator in US dollar terms (change in percent) & 34.2 & -24.5 & 14.1 & 10.5 & 18.6 & 23.3 & -0.8 & -4.2 & -6.2 & -3.9 & -6.6 & 0.3 & -0.7 & -1.7 & 0.2 \\
\hline Effective interest rate (percent) 5/ & 2.6 & 1.0 & 0.6 & 2.5 & 1.1 & 0.6 & 1.1 & 1.9 & 1.7 & 1.6 & 1.5 & 1.4 & 1.9 & 1.4 & 1.7 \\
\hline Growth of exports of G\&S (US dollar terms, in percent) & 31.7 & -22.4 & 48.3 & 16.2 & 25.8 & 35.0 & 2.3 & -1.8 & -7.2 & -1.8 & -13.6 & 2.2 & -0.5 & -0.8 & 1.2 \\
\hline Growth of imports of G\&S (US dollar terms, in percent) & 45.5 & -11.9 & 23.4 & 18.6 & 16.3 & 31.4 & -0.8 & -2.5 & 4.2 & 6.7 & 2.3 & 6.9 & 5.6 & -1.4 & 4.1 \\
\hline Grant element of new public sector borrowing (in percent) & & & & $\ldots$ & $\ldots$ & 33.4 & 36.8 & 36.9 & 36.9 & 36.9 & 36.9 & 36.3 & -8.1 & -8.1 & -2.1 \\
\hline Government revenues (excluding grants, in percent of GDP) & 46.6 & 29.2 & 37.8 & & & 52.1 & 53.6 & 53.6 & 53.1 & 53.0 & 51.4 & & 46.3 & 38.1 & 43.7 \\
\hline Aid flows (in Billions of US dollars) 7/ & 0.2 & 0.0 & 0.3 & & & 0.5 & 0.4 & 0.2 & 0.2 & 0.2 & 0.2 & & 0.1 & 0.1 & \\
\hline o/w Grants & 0.1 & 0.0 & 0.0 & & & 0.0 & 0.1 & 0.1 & 0.1 & 0.1 & 0.1 & & 0.1 & 0.1 & \\
\hline $\mathrm{o} / \mathrm{W}$ Concessional loans & 0.1 & 0.0 & 0.3 & & & 0.4 & 0.3 & 0.1 & 0.0 & 0.0 & 0.0 & & 0.0 & 0.0 & \\
\hline Grant-equivalent financing (in percent of GDP) 8/ & 0.1 & ... & $\ldots$ & & & 1.2 & 1.5 & 0.9 & 0.8 & 0.8 & 0.9 & & 0.6 & 0.3 & 0.5 \\
\hline Grant-equivalent financing (in percent of external financing) $8 /$ & $\ldots$ & $\ldots$ & $\ldots$ & & & 39.1 & 53.7 & 75.6 & 88.4 & 89.8 & 91.1 & & 79.0 & 68.2 & 76.7 \\
\hline \multicolumn{16}{|l|}{ Memorandum items: } \\
\hline Nominal GDP (Billions of US dollars) & 11.8 & 9.6 & 11.9 & & & 15.6 & 16.3 & 16.6 & 16.4 & 16.9 & 16.4 & & 21.4 & 35.0 & \\
\hline Nominal dollar GDP growth & 41.7 & -18.9 & 24.1 & & & 31.0 & 4.4 & 1.9 & -1.1 & 2.8 & -3.1 & 6.0 & 5.2 & 1.0 & 5.2 \\
\hline PV of PPG external debt (in Billions of US dollars) & & & 1.6 & & & 1.8 & 2.0 & 2.0 & 1.9 & 1.9 & 1.8 & & 1.3 & 0.6 & \\
\hline (PVt-PVt-1)/GDPt-1 (in percent) & & & & & & 2.1 & 0.9 & -0.1 & -0.3 & -0.3 & -0.3 & 0.3 & -0.4 & -0.2 & -0.3 \\
\hline Gross workers' remittances (Billions of US dollars) & 0.0 & 0.0 & 0.0 & & & 0.0 & 0.0 & 0.0 & 0.0 & 0.0 & 0.0 & & 0.0 & 0.0 & \\
\hline PV of PPG external debt (in percent of GDP + remittances) & 29.5 & 34.2 & 13.3 & & & 11.7 & 12.2 & 11.8 & 11.6 & 11.0 & 11.0 & & 6.2 & 1.8 & \\
\hline $\begin{array}{l}\text { PV of PPG external debt (in percent of exports + remittances) } \\
\text { (n) }\end{array}$ & 39.9 & 48.4 & 15.7 & & & 13.5 & 14.3 & 14.4 & 15.1 & 14.9 & 16.8 & & $\begin{array}{l}0.2 \\
12.0\end{array}$ & 4.9 & \\
\hline Debt service of PPG external debt (in percent of exports + remittances) & 29.5 & 34.2 & 1.3 & & & 0.8 & 0.9 & 1.1 & 1.2 & 1.1 & 1.3 & & 1.4 & 0.5 & \\
\hline
\end{tabular}

Sources: Country authorities; and staff estimates and projections.

$2 /$ Derived as $[r-g-\rho(1+g) /(1+g+\rho+g \rho)$ times previous period debt ratio, with $r=$ nominal interest rate; $g=$ real GDP growth rate, and $\rho=$ growth rate of GDP defflator in U.S. dollar terms. $3 /$ Includes exceptional financing (i.e., changes in arrears and debt relief); changes in gross foreign assets; and valuation adjustments. For projections also includes contribution from price and exchange rate changes 5/Current-yer interest payments divided byprivalent to is face value.

$6 /$ Historical averages and standard deviations are generally derived over the past 10 years, subject to data availability.

7/Defined as grants, concessional loans, and debt relief.

8/ Grant-equivalent financing includes grants provided directly to the government and through new borrowing (difference between the face value and the PV of new debt). 
Table 2. Republic of Congo: Sensitivity Analysis for Key Indicators of Public and Publicly Guaranteed External Debt, 2011-31 (In percent)

\begin{tabular}{|c|c|c|c|c|c|c|c|c|}
\hline & \multicolumn{8}{|c|}{ Projections } \\
\hline & 2011 & 2012 & 2013 & 2014 & 2015 & 2016 & 2021 & 2031 \\
\hline \multicolumn{9}{|c|}{ PV of debt-to-GDP ratio } \\
\hline Baseline & 12 & 12 & 12 & 12 & 11 & 11 & 6 & 2 \\
\hline \multicolumn{9}{|l|}{ A. Alternative Scenarios } \\
\hline A1. Key variables at their historical averages in 2011-31 1/ & 12 & 21 & 30 & 34 & 34 & 27 & -20 & -96 \\
\hline A2. New public sector loans on less favorable terms in $2011-312$ I & 12 & 13 & 12 & 12 & 12 & 12 & 7 & 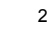 \\
\hline \multicolumn{9}{|l|}{ B. Bound Tests } \\
\hline B1. Real GDP growth at historical average minus one standard deviation in 2012-13 & 12 & 13 & 13 & 13 & 12 & 12 & 7 & 2 \\
\hline B2. Export value growth at historical average minus one standard deviation in 2012-13 3/ & 12 & 22 & 36 & 37 & 37 & 38 & 23 & 4 \\
\hline B3. US dollar GDP deflator at historical average minus one standard deviation in $2012-13$ & 12 & 13 & 13 & 13 & 12 & 12 & 7 & 2 \\
\hline B4. Net non-debt creating flows at historical average minus one standard deviation in 2012-13 4/ & 12 & 22 & 30 & 31 & 30 & 31 & 19 & 4 \\
\hline B5. Combination of B1-B4 using one-half standard deviation shocks & 12 & 17 & 16 & 16 & 15 & 15 & 9 & 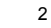 \\
\hline B6. One-time 30 percent nominal depreciation relative to the baseline in $20125 /$ & 12 & 17 & 17 & 17 & 16 & 16 & 9 & \\
\hline \multicolumn{9}{|c|}{ PV of debt-to-exports ratio } \\
\hline Baseline & 13 & 14 & 14 & 15 & 15 & 17 & 12 & 5 \\
\hline \multicolumn{9}{|l|}{ A. Alternative Scenarios } \\
\hline A1. Key variables at their historical averages in 2011-31 1/ & 13 & 24 & 36 & 44 & 46 & 41 & -40 & -262 \\
\hline A2. New public sector loans on less favorable terms in 2011-31 2/ & 13 & 15 & 15 & 16 & 16 & 18 & 13 & \\
\hline \multicolumn{9}{|l|}{ B. Bound Tests } \\
\hline B1. Real GDP growth at historical average minus one standard deviation in 2012-13 & 13 & 14 & 14 & 15 & 15 & 17 & 12 & 5 \\
\hline B2. Export value growth at historical average minus one standard deviation in 2012-13 3/ & 13 & 29 & 54 & 59 & 61 & 71 & 55 & 15 \\
\hline B3. US dollar GDP deflator at historical average minus one standard deviation in 2012-13 & 13 & 14 & 14 & 15 & 15 & 17 & 12 & 5 \\
\hline B4. Net non-debt creating flow s at historical average minus one standard deviation in 2012-13 4/ & 13 & 25 & 37 & 40 & 41 & 47 & 36 & 10 \\
\hline B5. Combination of B1-B4 using one-half standard deviation shocks & 13 & 19 & 19 & 20 & 20 & 22 & 16 & 6 \\
\hline B6. One-time 30 percent nominal depreciation relative to the baseline in $20125 /$ & 13 & 14 & 14 & 15 & 15 & 17 & 12 & 5 \\
\hline \multicolumn{9}{|c|}{ PV of debt-to-revenue ratio } \\
\hline Baseline & 23 & 23 & 22 & 22 & 21 & 21 & 13 & 5 \\
\hline \multicolumn{9}{|l|}{ A. Alternative Scenarios } \\
\hline A1. Key variables at their historical averages in 2011-31 1/ & 23 & 39 & 55 & 64 & 64 & 53 & -44 & -253 \\
\hline A2. New public sector loans on less favorable terms in 2011-31 2/ & 23 & 24 & 23 & 23 & 22 & 23 & 15 & 6 \\
\hline \multicolumn{9}{|l|}{ B. Bound Tests } \\
\hline B1. Real GDP growth at historical average minus one standard deviation in 2012-13 & 23 & 23 & 24 & 24 & 23 & 23 & 14 & 5 \\
\hline B2. Export value growth at historical average minus one standard deviation in 2012-13 3/ & 23 & 40 & 67 & 70 & 70 & 74 & 49 & 12 \\
\hline B3. US dollar GDP deflator at historical average minus one standard deviation in 2012-13 & 23 & 24 & 25 & 25 & 23 & 24 & 15 & 5 \\
\hline B4. Net non-debt creating flows at historical average minus one standard deviation in 2012-13 4/ & 23 & 40 & 56 & 58 & 58 & 61 & 40 & 10 \\
\hline B5. Combination of B1-B4 using one-half standard deviation shocks & 23 & 31 & 29 & 30 & 29 & 30 & 19 & 6 \\
\hline B6. One-time 30 percent nominal depreciation relative to the baseline in 20125 / & 23 & 32 & 32 & 31 & 30 & 31 & 19 & 7 \\
\hline \multicolumn{9}{|c|}{ Debt service-to-exports ratio } \\
\hline Baseline & 1 & 1 & 1 & 1 & 1 & 1 & 1 & 0 \\
\hline \multicolumn{9}{|l|}{ A. Alternative Scenarios } \\
\hline A1. Key variables at their historical averages in 2011-31 1/ & 1 & 1 & 1 & 2 & 2 & 3 & 1 & -17 \\
\hline A2. New public sector loans on less favorable terms in $2011-312 /$ & 1 & 1 & 1 & 1 & 1 & 1 & 1 & 0 \\
\hline \multicolumn{9}{|l|}{ B. Bound Tests } \\
\hline B1. Real GDP growth at historical average minus one standard deviation in $2012-13$ & 1 & 1 & 1 & 1 & 1 & 1 & 2 & 1 \\
\hline B2. Export value growth at historical average minus one standard deviation in $2012-133$ / & 1 & 1 & 2 & 3 & 3 & 5 & 7 & \\
\hline B3. US dollar GDP deflator at historical average minus one standard deviation in $2012-13$ & 1 & 1 & 1 & 1 & 1 & 1 & 2 & 1 \\
\hline B4. Net non-debt creating flows at historical average minus one standard deviation in 2012-13 4/ & 1 & 1 & 1 & 2 & 2 & 4 & 5 & 2 \\
\hline B5. Combination of B1-B4 using one-half standard deviation shocks & 1 & 1 & 1 & 1 & 1 & 2 & 2 & 1 \\
\hline B6. One-time 30 percent nominal depreciation relative to the baseline in 20125 / & 1 & 1 & 1 & 1 & 1 & 1 & 2 & 1 \\
\hline Debt service-to-revenu & & & & & & & & \\
\hline Baseline & 1 & 2 & 2 & 2 & 2 & 2 & 2 & 0 \\
\hline A. Alternative Scenarios & & & & & & & & \\
\hline A1. Key variables at their historical averages in $2011-311 /$ & 1 & 1 & 2 & 2 & 2 & 3 & 1 & -17 \\
\hline A2. New public sector loans on less favorable terms in 2011-31 2/ & 1 & 2 & 2 & 2 & 2 & 2 & 2 & 0 \\
\hline B. Bound Tests & & & & & & & & \\
\hline B1. Real GDP growth at historical average minus one standard deviation in 2012-13 & 1 & 2 & 2 & 2 & 2 & 2 & 2 & \\
\hline B2. Export value growth at historical average minus one standard deviation in $2012-13$ 3/ & 1 & 2 & 2 & 3 & 3 & 5 & 7 & 2 \\
\hline B3. US dollar GDP deflator at historical average minus one standard deviation in $2012-13$ & 1 & 2 & 2 & 2 & 2 & 2 & 2 & 1 \\
\hline B4. Net non-debt creating flows at historical average minus one standard deviation in 2012-13 4/ & 1 & 2 & 2 & 3 & 3 & 5 & 5 & 2 \\
\hline B5. Combination of B1-B4 using one-half standard deviation shocks & 1 & 2 & 2 & 2 & 2 & 3 & 3 & \\
\hline B6. One-time 30 percent nominal depreciation relative to the baseline in 20125 / & 1 & 2 & 2 & 2 & 2 & 2 & 3 & \\
\hline Memorandum item: & & & & & & & & \\
\hline Grant element assumed on residual financing (i.e., financing required above baseline) 6/ & 5 & 5 & 5 & 5 & 5 & 5 & 5 & 5 \\
\hline
\end{tabular}

Sources: Country authorities; and staff estimates and projections.

1/Variables include real GDP growth, growth of GDP deflator (in U.S. dollar terms), non-interest current account in percent of GDP, and non-debt creating flows.

2/ Assumes that the interest rate on new borrowing is by 2 percentage points higher than in the baseline., while grace and maturity periods are the same as in the baseline.

$3 /$ Exports values are assumed to remain permanently at the lower level, but the current account as a share of GDP is assumed to return to its baseline level after the

shock (implicitly assuming an offsetting adjustment in import levels)

$4 /$ Includes official and private transfers and FDI.

$5 /$ Depreciation is defined as percentage decline in dollar/local currency rate, such that it never exceeds 100 percent.

6/ Applies to all stress scenarios except for A2 (less favorable financing) in which the terms on all new financing are as specified in footnote 2 . 
Table 3. Republic of Congo: Public Sector Debt Sustainability Framework, Baseline Scenario, 2008-31

(In percent of GDP, unless otherwise indicated)

\begin{tabular}{|c|c|c|c|c|c|c|c|c|c|c|c|c|c|c|c|}
\hline & \multicolumn{3}{|c|}{ Actual } & \multirow[b]{2}{*}{ Average } & \multirow[b]{2}{*}{$\begin{array}{l}\text { Standard } \\
\text { Deviation }\end{array}$} & \multicolumn{4}{|l|}{ Estimate } & \multicolumn{3}{|c|}{ Projections } & \multirow[b]{2}{*}{2021} & \multirow[b]{2}{*}{2031} & \multirow[b]{2}{*}{$\begin{array}{l}2017-31 \\
\text { Average }\end{array}$} \\
\hline & 2008 & 2009 & 2010 & & & 2011 & 2012 & 2013 & 2014 & 2015 & 2016 & $\begin{array}{c}2011-16 \\
\text { Average }\end{array}$ & & & \\
\hline Public sector debt $1 /$ & 68.5 & 57.3 & 25.3 & & & 21.1 & 21.1 & 20.0 & 19.3 & 18.2 & 18.2 & & 9.9 & 2.4 & \\
\hline $\mathrm{o} / \mathrm{w}$ foreign-currency denominated & 59.7 & 50.2 & 22.7 & & & 19.6 & 20.2 & 19.5 & 19.2 & 18.2 & 18.2 & & 9.9 & 2.3 & \\
\hline Change in public sector debt & -30.1 & -11.1 & -32.0 & & & -4.2 & 0.0 & -1.0 & -0.8 & -1.1 & 0.0 & & -1.3 & -0.3 & \\
\hline Identified debt-creating flows & -52.9 & 0.2 & -48.7 & & & -37.1 & -33.0 & -31.7 & -28.2 & -27.0 & -19.4 & & -9.8 & -4.3 & \\
\hline Primary deficit & -25.1 & -5.7 & -15.4 & -13.8 & 7.1 & -31.2 & -32.3 & -31.5 & -28.6 & -26.6 & -20.1 & -28.4 & -9.3 & -4.3 & -7.8 \\
\hline Revenue and grants & 47.3 & 29.5 & 37.9 & & & 52.3 & 54.3 & 54.4 & 53.9 & 53.8 & 52.2 & & 46.9 & 38.4 & \\
\hline of which: grants & 0.7 & 0.3 & 0.1 & & & 0.3 & 0.7 & 0.7 & 0.8 & 0.8 & 0.9 & & 0.6 & 0.3 & \\
\hline Primary (noninterest) expenditure & 22.2 & 23.8 & 22.4 & & & 21.1 & 22.0 & 22.9 & 25.3 & 27.2 & 32.1 & & 37.6 & 34.1 & \\
\hline Automatic debt dynamics & -17.7 & 7.7 & -9.0 & & & -5.9 & -0.7 & -0.2 & 0.4 & -0.4 & 0.7 & & -0.4 & 0.0 & \\
\hline Contribution from interest rate/grow th differential & -7.8 & -2.4 & -5.8 & & & -2.0 & -1.2 & -1.3 & -1.2 & -1.4 & -0.8 & & -0.7 & -0.1 & \\
\hline of which: contribution from average real interest rate & -2.6 & 2.4 & -1.2 & & & -0.5 & -0.1 & -0.1 & -0.1 & -0.2 & -0.2 & & -0.1 & 0.0 & \\
\hline of which: contribution from real GDP growth & -5.2 & -4.8 & -4.6 & & & -1.5 & -1.1 & -1.3 & -1.0 & -1.2 & -0.7 & & -0.6 & -0.1 & \\
\hline Contribution from real exchange rate depreciation & -9.9 & 10.1 & -3.2 & & & -3.9 & 0.5 & 1.1 & 1.5 & 1.0 & 1.6 & & $\ldots$ & $\ldots$ & \\
\hline Other identified debt-creating flows & -10.1 & -1.7 & -24.3 & & & 0.0 & 0.0 & 0.0 & 0.0 & 0.0 & 0.0 & & 0.0 & 0.0 & \\
\hline Privatization receipts (negative) & 0.0 & 0.0 & 0.0 & & & 0.0 & 0.0 & 0.0 & 0.0 & 0.0 & 0.0 & & 0.0 & 0.0 & \\
\hline Recognition of implicit or contingent liabilities & 0.0 & 0.0 & 0.0 & & & 0.0 & 0.0 & 0.0 & 0.0 & 0.0 & 0.0 & & 0.0 & 0.0 & \\
\hline Debt relief (HIPC and other) & -10.1 & -1.7 & -24.3 & & & 0.0 & 0.0 & 0.0 & 0.0 & 0.0 & 0.0 & & 0.0 & 0.0 & \\
\hline Other (specify, e.g. bank recapitalization) & 0.0 & 0.0 & 0.0 & & & 0.0 & 0.0 & 0.0 & 0.0 & 0.0 & 0.0 & & 0.0 & 0.0 & \\
\hline Residual, including asset changes & 22.9 & -11.4 & 16.7 & & & 32.9 & 32.9 & 30.7 & 27.4 & 25.9 & 19.4 & & 8.4 & 4.0 & \\
\hline \multicolumn{16}{|l|}{ Other Sustainability Indicators } \\
\hline $\mathrm{PV}$ of public sector debt & $\ldots$ & $\ldots$ & 15.9 & & & 13.3 & 13.1 & 12.3 & 11.7 & 11.1 & 11.1 & & 6.2 & 1.8 & \\
\hline $\mathrm{o} / \mathrm{w}$ foreign-currency denominated & $\ldots$ & $\ldots$ & 13.3 & & & 11.7 & 12.2 & 11.8 & 11.6 & 11.0 & 11.0 & & 6.2 & 1.8 & \\
\hline o/w external & $\ldots$ & $\ldots$ & 13.3 & & & 11.7 & 12.2 & 11.8 & 11.6 & 11.0 & 11.0 & & 6.2 & 1.8 & \\
\hline PV of contingent liabilities (not included in public sector debt) & $\ldots$ & $\ldots$ & $\ldots$ & & & $\ldots$ & & & $\ldots$ & & $\ldots$ & & $\ldots$ & $\ldots$ & \\
\hline Gross financing need $2 /$ & -21.1 & -2.9 & -14.3 & & & -30.5 & -31.5 & -30.6 & -27.7 & -25.8 & -19.2 & & -8.6 & -4.1 & \\
\hline $\mathrm{PV}$ of public sector debt-to-revenue and grants ratio (in percent) & $\ldots$ & & 42.1 & & & 25.3 & 24.1 & 22.6 & 21.7 & 20.6 & 21.2 & & 13.2 & 4.7 & \\
\hline$P V$ of public sector debt-to-revenue ratio (in percent) & $\ldots$ & $\ldots$ & 42.1 & & & 25.4 & 24.4 & 22.9 & 22.0 & 20.9 & 21.6 & & 13.4 & 4.8 & \\
\hline o/w external $3 /$ & & & 35.1 & & & 22.5 & 22.7 & 22.1 & 21.9 & 20.8 & 21.5 & & 13.3 & 4.7 & \\
\hline Debt service-to-revenue and grants ratio (in percent) $4 /$ & 8.6 & 9.6 & 2.9 & & & 1.4 & 1.5 & 1.6 & 1.7 & 1.6 & 1.7 & & 1.6 & 0.5 & \\
\hline Debt service-to-revenue ratio (in percent) $4 /$ & 8.7 & 9.7 & 3.0 & & & 1.4 & 1.5 & 1.6 & 1.7 & 1.6 & 1.7 & & 1.6 & 0.5 & \\
\hline Primary deficit that stabilizes the debt-to-GDP ratio & 4.9 & 5.4 & 16.5 & & & -27.0 & -32.2 & -30.5 & -27.8 & -25.6 & -20.1 & & -8.0 & -4.0 & \\
\hline \multicolumn{16}{|l|}{ Key macroe conomic and fiscal assumptions $5 /$} \\
\hline Real GDP grow th (in percent) & 5.6 & 7.5 & 8.8 & 4.7 & 3.2 & 6.3 & 5.3 & 6.4 & 5.4 & 6.9 & 3.7 & 5.7 & 5.9 & 2.8 & 5.0 \\
\hline Average nominal interest rate on forex debt (in percent) & 2.3 & 1.1 & 0.7 & 3.1 & 2.4 & 0.8 & 0.8 & 0.8 & 0.8 & 0.8 & 0.8 & 0.8 & 1.0 & 0.5 & 0.9 \\
\hline Average real interest rate on domestic debt (in percent) & -18.7 & 28.0 & -16.2 & -1.3 & 21.5 & -16.3 & & $\ldots$ & $\ldots$ & & $\ldots$ & & $\ldots$ & $\ldots$ & $\ldots$ \\
\hline Real exchange rate depreciation (in percent, + indicates depreciation) & -12.5 & 18.1 & -6.9 & -5.0 & 15.3 & -18.2 & & & & & $\ldots$ & & $\ldots$ & $\ldots$ & $\ldots$ \\
\hline Inflation rate (GDP deflator, in percent) & 25.0 & -20.3 & 19.8 & 6.2 & 16.1 & 19.5 & -0.3 & -3.5 & -5.4 & -3.1 & -5.9 & 0.2 & -0.7 & 1.9 & 0.4 \\
\hline Grow th of real primary spending (deflated by GDP deflator, in percent) & -0.1 & 0.2 & 0.0 & 0.1 & 0.2 & 0.0 & 0.1 & 0.1 & 0.2 & 0.1 & 0.2 & 0.1 & 0.1 & 0.0 & 0.1 \\
\hline Grant element of new external borrowing (in percent) & $\ldots$ & $\ldots$ & $\ldots$ & & $\ldots$ & 33.4 & 36.8 & 36.9 & 36.9 & 36.9 & 36.9 & 36.3 & -8.1 & -8.1 & $\ldots$ \\
\hline
\end{tabular}

Sources: Country authorties; and staff estimates and projections.

$2 /$ Gross financing need is defined as the primary deficit plus debt service plus the stock of short-term debt at the end of the last period. $3 /$ Revenues excluding grants.

$4 /$ Debt service is defined as the sum of interest and amortization of medium and long-term debt.

$5 /$ Historical averages and standard deviations are generally derived over the past 10 years, subject to data availability 
Table 4. Republic of Congo: Sensitivity Analysis for Key Indicators of Public Debt 2011-31

\begin{tabular}{ll}
\hline & \\
\hline Baseline & PV of Debt-to-GDP Ratio
\end{tabular}

\section{A. Alternative scenarios}

A1. Real GDP growth and primary balance are at historical averages

A2. Primary balance is unchanged from 2011

A3. Permanently lower GDP growth 1/

Projections

\begin{tabular}{llllllll}
\hline 2011 & 2012 & 2013 & 2014 & 2015 & 2016 & 2021 & 2031 \\
\hline
\end{tabular}

\section{B. Bound tests}

B1. Real GDP growth is at historical average minus one standard deviations in 2012-2013

B2. Primary balance is at historical average minus one standard deviations in 2012-2013

B3. Combination of B1-B2 using one half standard deviation shocks

B4. One-time 30 percent real depreciation in 2012

B5. 10 percent of GDP increase in other debt-creating flows in 2012

\section{PV of Debt-to-Revenue Ratio 2/}

Baseline

$\begin{array}{llllllll}13 & 13 & 12 & 12 & 11 & 11 & 6 & 2\end{array}$

A. Alternative scenarios

A1. Real GDP growth and primary balance are at historical averages

A2. Primary balance is unchanged from 2011

A3. Permanently lower GDP growth 1

\begin{tabular}{|c|c|c|c|c|c|c|c|}
\hline 13 & 31 & 48 & 65 & 80 & 90 & 92 & -1 \\
\hline 13 & 14 & 14 & 10 & 5 & -6 & -92 & -327 \\
\hline 13 & 13 & 13 & 14 & 15 & 17 & 24 & 61 \\
\hline
\end{tabular}

\section{B. Bound tests}

B1. Real GDP growth is at historical average minus one standard deviations in 2012-2013

B2. Primary balance is at historical average minus one standard deviations in 2012-2013

$\mathrm{B} 3$. Combination of B1-B2 using one half standard deviation shocks

B4. One-time 30 percent real depreciation in 2012

B5. 10 percent of GDP increase in other debt-creating flows in 2012

Debt Service-to-Revenue Ratio 2/

Baseline

$\begin{array}{rrrrrrrr}13 & 15 & 20 & 24 & 28 & 33 & 46 & 70 \\ 13 & 37 & 61 & 63 & 62 & 66 & 56 & 45 \\ 13 & 35 & 58 & 62 & 64 & 71 & 73 & 80 \\ 13 & 18 & 17 & 17 & 16 & 17 & 12 & 7 \\ 13 & 23 & 22 & 22 & 21 & 22 & 16 & 10\end{array}$

$\begin{array}{llllllll}25 & 24 & 23 & 22 & 21 & 21 & 13 & 5\end{array}$

$\begin{array}{rrrrrrrr}25 & 57 & 89 & 120 & 148 & 173 & 195 & -2 \\ 25 & 26 & 25 & 19 & 10 & -11 & -195 & -851\end{array}$

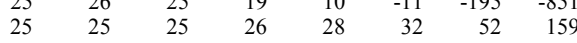

$\begin{array}{rrrrrrrr}25 & 28 & 37 & 45 & 52 & 64 & 99 & 181 \\ 25 & 69 & 112 & 116 & 116 & 126 & 120 & 116 \\ 25 & 64 & 106 & 115 & 120 & 136 & 155 & 207 \\ 25 & 33 & 31 & 31 & 30 & 32 & 25 & 19 \\ 25 & 42 & 40 & 41 & 40 & 42 & 35 & 27\end{array}$

\section{A. Alternative scenarios}

A1. Real GDP growth and primary balance are at historical averages

A2. Primary balance is unchanged from 2011

A3. Permanently lower GDP growth 1 /

$\begin{array}{rrrrrrrr}1 & 1 & 3 & 4 & 6 & 7 & 19 & 11 \\ 1 & 1 & 2 & 2 & 1 & 1 & -6 & -62 \\ 1 & 1 & 2 & 2 & 2 & 2 & 4 & 12\end{array}$

\section{B. Bound tests}

B1. Real GDP growth is at historical average minus one standard deviations in 2012-2013

B2. Primary balance is at historical average minus one standard deviations in 2012-2013

B3. Combination of B1-B2 using one half standard deviation shocks

B4. One-time 30 percent real depreciation in 2012

B5. 10 percent of GDP increase in other debt-creating flows in 2012

$\begin{array}{rrrrrrrr}1 & 2 & 2 & 2 & 3 & 3 & 7 & 16 \\ 1 & 1 & 3 & 5 & 5 & 5 & 12 & 13 \\ 1 & 2 & 3 & 5 & 5 & 6 & 14 & 21 \\ 1 & 2 & 2 & 2 & 2 & 3 & 3 & 2 \\ 1 & 1 & 2 & 2 & 2 & 2 & 4 & 3\end{array}$

Sources: Country authorities; and staff estimates and projections.

1/ Assumes that real GDP growth is at baseline minus one standard deviation divided by the square root of the length of the projection period.

2/ Revenues are defined inclusive of grants. 


\title{
INTERNATIONAL MONETARY FUND \\ REPUBLIC OF CONGO
}

Fifth and Sixth Reviews Under the Three-Year Arrangement Under the Extended Credit Facility and Financing Assurances Review

\author{
Informational Annex \\ Prepared by the African Department \\ (In consultation with other departments) \\ Approved by Domenico Fanizza and Dhaneshwar Ghura
}

July 15, 2011

Contents

Annexes

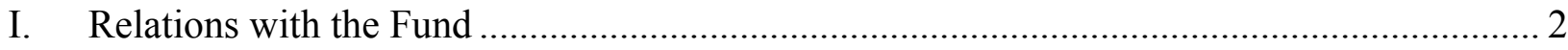

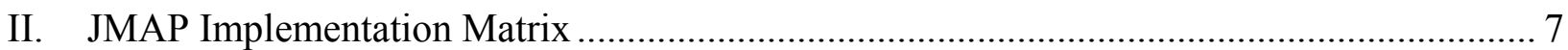

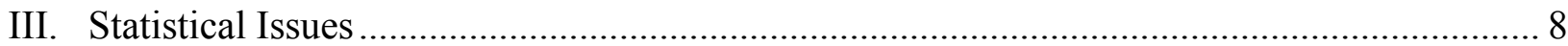


Annex I-Republic of Congo: Relations with the Fund (As of May 31, 2011)

I. Membership Status: Joined: 07/10/1963; Article VIII

II. General Resources Account:

Quota

Fund holdings of currency

Reserve tranche position

$\underline{\text { SDR Million }}$

84.60

$\underline{\text { Percent Quota }}$

84.03

100.00

0.58

99.33

0.68

III. SDR Department:

Net cumulative allocation

$\underline{\text { SDR Million }}$

$\underline{\text { Percent Allocation }}$

Holdings

79.69

100.00

70.05

87.90

IV. Outstanding Purchases and Loans:

$\underline{\text { SDR Million }}$

$\underline{\text { Percent Quota }}$

ECF arrangements

18.69

22.10

V. Latest Financial Arrangements:

$\begin{array}{cccrr}\text { Type } & \begin{array}{c}\text { Approval } \\ \text { Date }\end{array} & \begin{array}{c}\text { Expiration } \\ \text { Date }\end{array} & \begin{array}{c}\text { Amount } \\ \text { Approved } \\ \text { (SDR Million) }\end{array} & \begin{array}{c}\text { Amount } \\ \text { Drawn } \\ \text { (SDR Million) }\end{array} \\ \text { ECF }^{1} & 12 / 08 / 2008 & 12 / 07 / 2011 & 8.46 & 6.04 \\ \text { ECF }^{1} & 12 / 06 / 2004 & 06 / 05 / 2008 & 54.99 & 23.58 \\ \text { ECF }^{1} & 06 / 28 / 1996 & 06 / 27 / 1999 & 69.48 & 13.90 \\ { }^{1} \text { Formerly PRGF } & & & & \end{array}$

VI. Projected Payments to Fund: (without HIPC assistance)

(SDR million; based on existing use of resources and present holdings of SDRs):

\begin{tabular}{|c|c|c|c|c|c|}
\hline & & & orthco & & \\
\hline & $\underline{2011}$ & 2012 & $\underline{2013}$ & 2014 & $\underline{2015}$ \\
\hline Principal & & 1.65 & 3.14 & 3.51 & 3.87 \\
\hline Charges/interest & $\underline{0.03}$ & $\underline{0.10}$ & $\underline{0.09}$ & $\underline{0.08}$ & $\underline{0.07}$ \\
\hline Total & 0.03 & 1.74 & 3.23 & 3.59 & 3.94 \\
\hline
\end{tabular}




\section{Implementation of HIPC Initiative:}

The Republic of Congo reached the completion point under the enhanced HIPC Initiative in January 2010.

I. Commitment of HIPC assistance

Enhanced Framework

Decision point date

March 2006

Assistance committed

by all creditors (US\$ million) ${ }^{1}$

Of which: IMF assistance (US\$ million)

(SDR equivalent in millions)

Completion point date

January 2010

II. Disbursement of IMF assistance (SDR million)

Assistance disbursed to the member

Interim assistance

Completion point balance

Additional disbursement of interest income ${ }^{2}$

0.86

III. Implementation of Multilateral Debt Relief Initiative (MDRI)

6.26

\section{Implementation of Multilateral Debt Relief Initiative (MDRI):}

I. $\quad$ MDRI-eligible debt (SDR Million) ${ }^{3} \quad 7.86$

Financed by: MDRI Trust $\quad 4.79$

Remaining HIPC resources $\quad 3.07$

II. Debt Relief by Facility (SDR Million)

Eligible Debt

\section{Delivery}

Date

January 2010

(1)

$\underline{\text { GRA }}$

N/A
PRGT

7.86 $\underline{\text { Total }}$

7.86

\footnotetext{
${ }^{1}$ Assistance committed under the original framework is expressed in net present value (NPV) terms at the completion point, and assistance committed under the enhanced framework is expressed in NPV terms at the decision point. Hence these two amounts can not be added.

${ }^{2}$ Under the enhanced framework, an additional disbursement is made at the completion point corresponding to interest income earned on the amount committed at the decision point but not disbursed during the interim period.

${ }^{3}$ The MDRI provides 100 percent debt relief to eligible member countries that qualified for the assistance. Grant assistance from the MDRI Trust and HIPC resources provide debt relief to cover the full stock of debt owed to the Fund as of end-2004 that remains outstanding at the time the member qualifies for such debt relief.
} 


\section{Safeguards Assessments:}

The Bank of the Central African States (BEAC) is the regional central bank of the Central African States. The most recent safeguards assessment of the BEAC was completed on July 6, 2009. The findings of this assessment indicate that implementation of previous safeguards recommendations on financial reporting, internal audit, and internal control has been limited, and that the changing risk profile of the BEAC foreign exchange holdings requires further actions to strengthen safeguards at the BEAC. Subsequent to revelation of Paris office fraud, a series of initial measures and longer term safeguard measures were agreed between IMF and BEAC in order to continue with country programs. In December 2009, BEAC adopted an action plan with the aim of reforming its own governance, and strengthening key safeguards. While implementation of the 2009 action plan is still in progress, BEAC adopted additional measures to address the weaknesses highlighted by the first special audit.

\section{Exchange Rate Arrangement:}

Congo's currency is the CFA franc, which is pegged to the Euro at a fixed rate of CFAF $655.957=$ Euro 1. Congo does not impose any restrictions on the making of payments and transfers for current international transactions.

\section{Article IV Consultations:}

Consultations with Congo are on a 24-month cycle, based on the Board decision on consultation cycles in program countries. The last Article IV consultation discussions and the Fourth Review under the Extended Credit Facility (ECF) arrangement were held in Brazzaville on November 9-23, 2010. The staff report (www.imf.org) was considered by the Executive Board on January 19, 2011.

\section{FSAP Participation : N/A.}


XIII. Technical Assistance :

\begin{tabular}{|c|c|c|c|}
\hline Subject & Department & Staff Member & Date \\
\hline Tax policy reform & FAD & Mario Mansour (head) & February 2011 \\
\hline Improving budget process & AFRITAC & $\begin{array}{l}\text { Abdoulahi Mfoubout } \\
\text { (head) }\end{array}$ & February 2011 \\
\hline National Accounts & AFRITAC & Hubert Gbossa & January 2011 \\
\hline National Accounts & AFRITAC & Maxime Bonkoungou & October 2010 \\
\hline National Accounts & AFRITAC & Huber Gbossa & April 2010 \\
\hline $\begin{array}{l}\text { Poverty-reducing spending } \\
\text { definition }\end{array}$ & AFRITAC & Jean Pierre Nuenang & March 2010 \\
\hline $\begin{array}{l}\text { Tax administration. } \\
\text { Strengthening capacity at } \\
\text { domestic revenue authority }\end{array}$ & AFRITAC & Philippe Laurent & February 2010 \\
\hline Customs administration & AFRITAC & Ives Soler & December 2009 \\
\hline Tax policy & FAD & Mario Mansur & Aug/Sept. 2009 \\
\hline $\begin{array}{l}\text { Assist the National Accounts } \\
\text { Team in finalizing the analysis of } \\
\text { main aggregates of } 2006 \text { NA } \\
\text { estimates }\end{array}$ & STA & $\begin{array}{l}\text { Mr. Gbossa (head), } \\
\text { AFRITAC C }\end{array}$ & Aug/Sept. 2009 \\
\hline $\begin{array}{l}\text { Assess the country's domestic } \\
\text { tax system and tax policy }\end{array}$ & $\begin{array}{l}\text { FAD and } \\
\text { AFRITAC } \\
\text { West }\end{array}$ & $\begin{array}{l}\text { Messrs. Montagnat- } \\
\text { Rentier (head) }\end{array}$ & July 2009 \\
\hline $\begin{array}{l}\text { Assist National Accounts Team } \\
\text { with analysis of main aggregates } \\
\text { of } 2006 \text { NA estimates and train } \\
\text { NA team in synthesis of accounts }\end{array}$ & STA & $\begin{array}{l}\text { Mr. Gbossa (head), } \\
\text { AFRITAC C }\end{array}$ & April 2009 \\
\hline $\begin{array}{l}\text { Follow-up on reforms for the } \\
\text { modernization of tax and } \\
\text { customs administrations } \\
\text { Identifying strategic priorities for } \\
\text { the next 3-4 years }\end{array}$ & $\begin{array}{l}\text { FAD and } \\
\text { AFRITAC } \\
\text { West }\end{array}$ & $\begin{array}{l}\text { Messrs. Montagnat- } \\
\text { Rentier, Lefebvre and } \\
\text { Koukpaizan }\end{array}$ & March 2009 \\
\hline $\begin{array}{l}\text { Follow-up mission on the } \\
\text { implementation of statistics TA } \\
\text { programs }\end{array}$ & AFRITAC C & Mr. Bonkoungou & March 2009 \\
\hline $\begin{array}{l}\text { Processing and monitoring of } \\
\text { fiscal information }\end{array}$ & AFRITAC C & Mr. Penanje & August 2008 \\
\hline Budget functional classification & AFRITAC C & Mrs. Lokpe and Nguenan & July 2007 \\
\hline $\begin{array}{l}\text { Poverty and Social Impact } \\
\text { Analysis }\end{array}$ & FAD & $\begin{array}{l}\text { Mr. Gillingham, Ms. } \\
\text { Lacoche, Mr. Manning }\end{array}$ & November 2007 \\
\hline Public expenditure management & FAD & Mr. Bouley & August 2006 \\
\hline Resident advisor on real sector & STA & Mr. Gbossa & Since February 2006 \\
\hline
\end{tabular}




\begin{tabular}{|l|l|l|l|}
\hline Subject & Department & Staff Member & Date \\
\hline $\begin{array}{l}\text { Modernization of tax and } \\
\text { customs administrations }\end{array}$ & FAD & $\begin{array}{l}\text { Messrs. Montagnat- } \\
\text { Rentier, Lesprit and Boilil }\end{array}$ & February 2004 \\
\hline Follow-up mission & FAD & Mr. Lepage & November 2003 \\
\hline Budget functional classification & FAD & $\begin{array}{l}\text { Messrs. Bouley, } \\
\text { Helis, and Lepage }\end{array}$ & October 2003 \\
\hline $\begin{array}{l}\text { Balance of payments statistics } \\
\text { Multisector statistics }\end{array}$ & $\begin{array}{l}\text { STA } \\
\text { STA }\end{array}$ & $\begin{array}{l}\text { Mr. Dessart } \\
\text { Messrs. Marie, Maiga, } \\
\text { and Mmes. Fisher, Matei, } \\
\text { Razin, and Balvani }\end{array}$ & $\begin{array}{l}\text { February 2007 } \\
\text { May 2002 }\end{array}$ \\
\hline Resident expert on statistics & STA & Mr. Metreau & Since October 2001 \\
\hline Balance of payments & STA & Mr. Fiévet & June 2001 \\
\hline Budget, tax, and customs & FAD & $\begin{array}{l}\text { Messrs. Bouley, Moussa, } \\
\text { Brik, and Mrs. Tricoire }\end{array}$ & April-May 2001 \\
\hline Resident tax expert & FAD & Mr. Laurent & 1995-97 \\
\hline Tax administration & FAD & Mr. Grandcolas & Nov. 1995-Apr. 1996 \\
\hline Tax administration & FAD & $\begin{array}{l}\text { Messrs. Grandcolas and } \\
\text { Castro }\end{array}$ & November 1994 \\
\hline
\end{tabular}

\section{Resident Representative:}

The resident representative, Mr. Oscar Edgardo Melhado Orellana, took up his assignment in September 2009. 
Annex II-Republic of Congo: JMAP Implementation Matrix

\begin{tabular}{|c|c|c|c|}
\hline Title & Products & Mission timing & Expected delivery \\
\hline \multicolumn{4}{|c|}{ A. Mutual information on relevant work programs } \\
\hline $\begin{array}{l}\text { Bank work program in } \\
\text { next } 12 \text { months }\end{array}$ & $\begin{array}{l}\text { Dialogue on Governance } \\
\text { AAA } \\
>\text { Statistical capacity building } \\
>\text { Employment and Growth } \\
\text { Study } \\
\text { New Lending: } \\
\text { D Governance and } \\
\text { Transparency Project - II } \\
\text { Regional } \\
\text { Telecommunication Project } \\
\text { Forestry management } \\
\text { project (TA) }\end{array}$ & $\begin{array}{l}\text { FY11 } \\
\text { Ongoing } \\
\text { March } 2011 \\
\text { March } 2011 \\
2011 \\
2011\end{array}$ & $\begin{array}{l}\text { Ongoing } \\
\text { Ongoing } \\
\text { June } 2011 \\
\text { August } 2011 \\
\text { July } 2011 \\
\text { March } 2012\end{array}$ \\
\hline \multirow{2}{*}{$\begin{array}{l}\text { IMF work program in } \\
\text { next } 12 \text { months }\end{array}$} & $\begin{array}{l}\text { Fifth and Sixth reviews under } \\
\text { the ECF arrangement }\end{array}$ & May/June 2011 & July 2011 \\
\hline & Article IV Consultations & March 2012 & April 2012 \\
\hline \multicolumn{4}{|c|}{ B. Requests for work program inputs } \\
\hline Fund request to Bank & $\begin{array}{l}\text { Preliminary study on } \\
\text { employment and growth }\end{array}$ & March 2011 & June 2011 \\
\hline \multirow{2}{*}{ Bank request to Fund } & $\begin{array}{l}\text { Macroeconomic updates for } \\
\text { program documents }\end{array}$ & FY11 and FY 12 & FY11 and FY 12 \\
\hline & $\begin{array}{l}\text { Support on taxation issues } \\
\text { related to the forestry sector }\end{array}$ & & FY 12 \\
\hline \multicolumn{4}{|c|}{ C. Agreement on joint products and missions } \\
\hline & $\begin{array}{l}\text { Collaboration on data on non- } \\
\text { oil sector growth }\end{array}$ & FY11 and FY 12 & FY11 and FY 12 \\
\hline
\end{tabular}




\section{Annex III}

\begin{tabular}{|l|}
\hline \multicolumn{1}{|c|}{$\begin{array}{c}\text { Republic of Congo-STATISTICAL ISSUES } \\
\text { As of July 1, 2011 }\end{array}$} \\
\hline \multicolumn{1}{|c|}{ I. Assessment of Data Adequacy for Surveillance } \\
\hline $\begin{array}{l}\text { General: Data provision has some shortcomings, but is broadly adequate for surveillance. The limited statistics } \\
\text { coverage and long delays in the provision of national accounts and balance of payments statistics are } \\
\text { particularly problematic. Since the occurrence of governance problems at the Bank of Central African States } \\
\text { (BEAC), timely delivery of financial sector data has been hampered. Poor coordination among different } \\
\text { governmental agencies is also an issue. Technical assistance has resulted in some progress but there is still need } \\
\text { to consolidate these gains and further strengthening of statistical capacities. }\end{array}$
\end{tabular}

National Accounts: National accounts data is weak, with inconsistencies, both internally and with balance of payments statistics. The Directorate General of Statistics (DGS) of the Ministry of Finance (MoF) provides Fund missions with a breakdown of GDP by expenditures and sectors, both in nominal and real terms. In the framework of moving to the SNA93 system of accounts, the DGS completed the national accounts estimates for 2005 (the new base year) and for 2006-07, with the assistance of the resident statistical expert and Central AFRITAC. Authorities are currently working with the Central AFRITAC to revise the national accounts' estimates back to 2000 and up to 2008. Annual data on employment in the central government are available from the MoF, but are not consistent with data from the civil service roster of the Ministry of the Civil Service. As part of the structural reforms undertaken in the context of the post-conflict program, the government completed an audit of public service employees, but the two civil service's databases are yet to be unified.

Price statistics: Movements in the prices of commodities consumed by households are recorded for the capital city of Brazzaville and for Pointe Noire - the second largest city. Data are compiled on a monthly basis. Starting August 2010, the authorities introduced a new CPI measure with new weights and a new composition of the basket of good and service. This new CPI is harmonized with that of other countries in the Central African Economic and Monetary Community (CEMAC).

Government finance statistics (GFS): Progress is underway to increase the comprehensiveness of the data through the adoption of systematic compilation practices and steps have been taken to computerize expenditure chain accounting. The Caisse Congolaise d'Amortissement (CCA) produces comprehensive data on the outstanding stock of external public debt, including arrears and their composition, together with detailed projections on debt service due. These data are provided to Fund missions. However, the debt-stock data cannot be reconciled with flow data in the balance of payments. The CCA also produces domestic debt data. Concerns still exist with regard to the reconciliation of fiscal and monetary statistics. There is no centralized, comprehensive database on the operations of public enterprises. However, some information has been made available to Fund missions by individual enterprises. TOFE compilers do not have access to relevant financial statements of the Société Nationale des Pétroles Congolais (SNPC), which carries out several operations on behalf of the government (notably in the oil sector).

Monetary statistics: BEAC regularly reports monetary, interest rates, and exchange rate statistics for publication in IFS, but delays are increasing (currently 3 months). In 2007, the BEAC started a project to migrate monetary statistics of member countries of the CEMAC to the methodology in the Monetary and Financial Statistics Manual (MFSM). As a part of this project, a regional workshop was organized by the BEAC 
in December 2007 to finalize the mapping of source data from commercial banks to the MFSM concepts and framework. STA participated in this workshop to provide guidance and advice. Submission of data by the BEAC using the standardized report forms is pending. Following governance issues occurred in 2009 at the BEAC data delivery on financial system indicators has been hampered.

The accuracy of national monetary statistics may be affected by large cross-border movements of BEAC issued banknotes among CEMAC member countries. However, the Republic of Congo is moderately affected by such movements: 6.2 percent of banknotes issued in the Republic of Congo by the BEAC national directorate circulate in Cameroon and 1.2 percent in Gabon, while currency in circulation in the Republic of Congo includes 2.3 percent of banknotes from Cameroon and 3.2 percent from Gabon. The magnitude of banknote movements between the Republic of Congo and CEMAC member countries other than Cameroon and Gabon is very small.

Balance of payments: Although BEAC produces balance of payments data, delays occur and data suffers from significant inconsistencies with other datasets. The opacity surrounding certain transactions in the oil sector introduces an extra layer of imprecision. In addition, net investment flows are overestimated by significant unrecorded disinvestment operations that are part of the tax regime arrangements obtained by nonresident oildrilling companies. Balance of payments data for 1995 through 2005 are published in the Fund's International Financial Statistics (IFS), and STA is working with the authorities in updating the data series. In February 2007, a follow-up STA balance of payments statistics mission made a number of recommendations aimed at improving institutional arrangements for balance of payments statistics compilation and validation. Progress in this regard has been slow, consequently delaying the process for finalizing BOP data for 2005-08.

\section{Data Standards and Quality}

The Republic of Congo participates in the General No data ROSC is available.

Data Dissemination System (GDDS) since November

5, 2003. However, the metadata posted on the Fund's

Dissemination Standards Bulletin Board (DSBB) has not been updated or certified since 2003.

\section{Reporting to STA}

The Republic of Congo has reported (in September 2008) GFS data to STA for fiscal years 2004 and 2005 using the GFSM 2001 template - data for 2006 and 2007 has experienced delays. High-frequency fiscal data has not yet been reported for publication in IFS. The compilation of GFS statistics to be reported to the Fund should be carried out in close cooperation with the division in charge of TOFE statistics. 


\section{REPUblic OF CONGO: TABLE OF COMMON INDICATORS REQUIRED FOR SURVEILLANCE As of July 1, 2011}

\begin{tabular}{|c|c|c|c|c|c|}
\hline & $\begin{array}{l}\text { Date of latest } \\
\text { observation }\end{array}$ & $\begin{array}{l}\text { Date } \\
\text { received }\end{array}$ & $\begin{array}{c}\text { Frequency } \\
\text { of } \\
\text { Data }^{7}\end{array}$ & $\begin{array}{l}\text { Frequency } \\
\quad \text { of } \\
\text { Reporting }^{7}\end{array}$ & $\begin{array}{l}\text { Frequency of } \\
\text { publication }^{7}\end{array}$ \\
\hline Exchange Rates & Current & Current & $\mathrm{D}$ & $\mathrm{D}$ & $\mathrm{D}$ \\
\hline $\begin{array}{l}\text { International Reserve Assets and Reserve } \\
\text { Liabilities of the Monetary Authorities }{ }^{1}\end{array}$ & March 2011 & June 2011 & M & M & M \\
\hline Reserve/Base Money & March 2011 & June 2011 & M & M & M \\
\hline Broad Money & March 2011 & June 2011 & M & M & M \\
\hline Central Bank Balance Sheet & March 2011 & June 2011 & M & M & M \\
\hline Consolidated Balance Sheet of the Banking System & March 2011 & June 2011 & M & M & M \\
\hline Interest Rates $^{2}$ & March 2011 & June 2011 & M & M & M \\
\hline Consumer Price Index & May 2011 & June 2011 & M & M & M \\
\hline $\begin{array}{l}\text { Revenue, Expenditure, Balance and Composition of } \\
\text { Financing }{ }^{3} \text { - General Government }\end{array}$ & N/A & N/A & N/A & $\mathrm{N} / \mathrm{A}$ & $\mathrm{N} / \mathrm{A}$ \\
\hline $\begin{array}{l}\text { Revenue, Expenditure, Balance and Composition of } \\
\text { Financing }{ }^{3} \text { - Central Government }\end{array}$ & March 2011 & June 2011 & $\mathrm{M}$ & M & Q \\
\hline $\begin{array}{l}\text { Stocks of Central Government and Central } \\
\text { Government-Guaranteed Debt }\end{array}$ & March 2011 & June 2011 & Q & Q & Q \\
\hline External Current Account Balance & December 2010 & Mar. 2011 & A & A & A \\
\hline Exports and Imports of Goods and Services & December 2010 & Mar. 2011 & A & A & A \\
\hline GDP/GNP & December 2010 & Mar. 2011 & A & A & A \\
\hline Gross External Debt & March 2011 & June 2011 & Q & Q & Q \\
\hline International Investment Position ${ }^{6}$ & $\ldots$ & $\ldots$ & NA & NA & NA \\
\hline
\end{tabular}

\footnotetext{
${ }^{1}$ Includes reserve assets pledged or otherwise encumbered as well as net derivative positions.

${ }^{2}$ Both market-based and officially-determined, including discount rates, money market rates, rates on treasury bills, notes and bonds.

${ }^{3}$ Foreign, domestic bank, and domestic non-bank financing.

${ }^{4}$ The general government consists of the central government (budgetary funds, extra budgetary funds, and social security funds) and state and local governments.

${ }^{5}$ Including currency and maturity composition.

${ }^{6}$ Includes external gross financial asset and liability positions vis-à-vis nonresidents.

${ }^{7}$ Daily (D), Weekly (W), Monthly (M), Quarterly (Q), Annually (A); Irregular (I); Not Available (NA).
} 


\section{Statement by the IMF Staff Representative July 27, 2011}

1. This statement contains information made available since the staff report was sent for circulation regarding un-repatriated oil receipts held by the government of the Republic of Congo outside of the regional central bank (BEAC) which provides clarification of financing items contained in Tables $3 \mathrm{a}$ and $3 \mathrm{~b}$ of the staff report. The information contained herein does not change the thrust of the staff appraisal, and confirms the satisfactory program performance to date.

\section{Background}

2. In the course of reviewing budget financing, the Congolese authorities informed staff of un-repatriated oil receipts from oil sales starting in January 2010 held in a bank offshore, in breach of the BEAC statutes. They reported that they took the decision to delay repatriation of a portion of their oil receipts in response to (a) the fraud at BEAC discovered in late-2009; and (b) the severe weaknesses uncovered in the May 2010 BEAC audit report.

3. While accumulating a portion of oil receipts offshore, Congo also increased its reserve holdings in BEAC. At end-March 2011, Congo's holdings of (imputed) liquid foreign exchange reserves in BEAC amounted to 38 percent of 2010 GDP; accounted for over onethird of regional reserve holdings; and stood at about 150 percent of broad money.

4. The authorities confirmed that there have been no transfers of reserves from the regional central bank or other oil proceeds into the offshore account, and that the funds in question are not encumbered.

\section{Repatriation}

5. In April 2011, the Congolese authorities began repatriating the oil receipts held offshore. As of end-June, they had repatriated about a third of the end-March stock, and have committed to repatriating the remaining oil receipts by end-2011, and to comply with BEAC statutes regarding current and future foreign exchange receipts.

6. On July 12, 2011, the Congolese authorities informed the BEAC Board of both the offshore holdings and the timetable for repatriation, and encouraged all countries to repatriate offshore reserve holdings. In response, the BEAC Board formed a committee to study the pooling of reserves by member countries.

\section{Impact on the fifth and sixth program reviews}

7. The existence of un-repatriated oil receipts which are held abroad does not impact any performance criteria under the ECF arrangement. These offshore holdings do explain the difference between the programmed indicative target on net domestic financing of the 
government and the lower accumulation of deposits in the BEAC, thereby confirming that fiscal performance remained in line with the thrust of the program.

8. The fifth review was delayed pending further clarification of financing flows first brought to light at the time of the fourth review. For the purposes of program monitoring, the authorities have provided stock data on un-repatriated oil receipts held as deposits in offshore accounts, and staff has recorded these flows as "Other" foreign financing in the fiscal tables (Tables $3 a$ and $3 b$ ) of the staff report for the fifth and sixth (final) reviews.

\section{The safeguards policy}

9. The authorities report that the offshore deposits are subject to the same treatment as other budget operations, and are classified in budget reports to parliament as part of the stabilization account. These deposits, however, were not subject to safeguards assessment.

10. Informing the BEAC Board that foreign assets were not repatriated within the timeframe prescribed by BEAC regulations was an important first step toward bringing the Republic of Congo into full compliance with BEAC law. Beginning to repatriate the deposits supports the authorities' stated commitment to full regularization. These initial steps address the particular safeguard concern that lack of transparency on repatriation could have adverse implications for broader governance reforms, which will be key to success in strengthening safeguards at the BEAC. 


\section{IMF Executive Board Completes Fifth and Sixth Reviews Under the Extended Credit Facility Arrangement for the Republic of Congo and Approves US\$3.9 Million Disbursement}

The Executive Board of the International Monetary Fund (IMF) approved on July 27 the fifth and sixth reviews of the Republic of the Congo's economic program supported by the Extended Credit Facility (ECF). The approval will enables an immediate disbursement in an amount equivalent to SDR 2.42 million (about US\$3.9 million), bringing total disbursements under the arrangement to an amount equivalent to SDR 8.46 million (about US\$13.6 million).

The ECF Arrangement for the Republic of Congo in the amount of SDR 8.46 million (10 percent of quota was approved by the Executive Board on December 8, 2008 (See Press Release No. 08/311). The ECF supported program will expire with the completion of the sixth and final review.

Following the Board discussion of the Republic of Congo, Ms. Nemat Shafik, Deputy Managing Director and Acting Chair, issued the following statement:

"The authorities are to be commended for their satisfactory implementation of the ECFsupported program. The outlook is favorable, supported by strong polices and improving external conditions. At the same time, progress on key structural reforms has built resilience to shocks. The scaling up of public investment and the structural reforms in train bode well for raising nonoil growth and durable poverty reduction.

"Reform momentum should be maintained to build on the progress achieved under the program. Increased focus should be placed on strengthening policymaking and building institutions. Reforms under way in the fiscal area will help reduce the complexity of the tax system and raise nonoil revenue. 
"Implementation of the recently-adopted action plan to improve the business climate will complement the ongoing public infrastructure investment. Increased coordination among ministries, donors, and consultants will be important in moving the reform agenda forward.

"Congo's external position has improved markedly following the HIPC Initiative completion point. It will be important to safeguard this gain by improving debt and oil wealth management. Debt relief agreements with bilateral and commercial creditors should be finalized.

“The authorities' commitment to repatriate export receipts to the BEAC is welcome. The authorities are encouraged to continue their efforts to enhance the potential of the regional economic and monetary community," Mrs. Shafik added. 


\section{Statement by Kossi Assimaidou, Executive Director for the Republic of Congo July 27, 2011}

\section{Introduction}

On behalf of my Congolese authorities, I would like to thank staff for the constructive policy dialogue during the recent missions held in Brazzaville in March and May-June 2011.

The Republic of Congo's implementation of the ECF-supported program continues to be satisfactory. The authorities met all quantitative targets at end-December 2010 and endMarch 2011, including the floor on the basic non-oil primary fiscal balance despite spending pressures in 2010. They also observed all structural benchmarks - though some with delaynotably the benchmark on the implementation of the new procurement code which ultimately exceeded the program threshold. My Congolese authorities will continue their efforts to strengthen governance in the oil sector, as can be seen through the observance of all structural benchmarks and the realization of meaningful progress towards EITI compliance.

Throughout the program, the authorities have maintained the momentum of economic reforms and have kept the fight against poverty at the core of their development agenda. An important goal is to close the country's immense infrastructure gap, including by bringing water and electricity to the main urban centers. Another key objective of the investments underway is to promote the diversification of the economy away from oil production which is expected to peak next year, while encouraging job creation in the private sector.

The Republic of Congo, which currently chairs the ministerial committee of the Central African Economic and Monetary Community (CEMAC) and the Board of Directors of the Community's central bank, BEAC, has reiterated its commitment to strengthen regional integration and address weaknesses at the regional institutions. Regarding more specifically the issue of the repatriation of foreign exchange receipts at the BEAC by member countries, I would like to underscore that my authorities take this issue very seriously. Accordingly, following a BEAC Board meeting held in Cameroun earlier this month, they have announced that they will comply with all obligations under BEAC rules. I would like to inform the Board that they have already initiated repatriation of part of these receipts, and that the repatriation of the remaining receipts held abroad should be finalized by the end of the year.

\section{Recent Economic Developments}

Congo's macroeconomic performance in 2010 was robust and broad-based. While growth in the oil sector remained strong, non-oil real GDP growth continued to increase and is projected to reach double-digit rates by 2013 - a sign of increased economic diversification that the investment program underway is intended to further enhance. 
Higher oil prices have helped shift the current account balance from a large deficit of 8.2 percent of GDP in 2009 to a comfortable surplus of 4.6 percent of GDP in 2010. Inflation, on average, remained stable compared to last year, at around 5 percent, although slightly above the CEMAC's convergence criterion of 3 percent.

The authorities have also successfully narrowed the basic non-oil primary deficit balance to 34.4 percent of GDP in 2010 from 44.3 percent in 2008 thanks to efforts on both revenuesthrough tax and customs administration reforms, tax policy changes - and expenditure management. In particular, the improved public financial management is yielding positive results.

\section{Program Implementation Going Forward}

The adjustment in the projected basic non-oil primary fiscal balance at end-December 2011 of 34.4 percent of non-oil GDP represents, over the three-year duration of the ECF-supported program, an improvement equivalent to 10 percent of non-oil GDP. The size of the fiscal consolidation corresponds to the amount envisioned at the time of the approval of the arrangement in December 2008.

Building on the progress made thus far, my authorities are determined to continue their fiscal consolidation efforts by taking decisive measures to further create fiscal space and enable a substantial increase in development spending. They intend thus to accelerate the diversification of the sources of revenue away from oil while making further efforts to reduce non-essential current expenditures. In this respect, they welcome the valuable recommendations made by recent FAD missions in Brazzaville. Based on the findings and recommendations of these missions, they have initiated an ambitious medium-term fiscal reform plan to help strengthen revenue mobilization, including through the elimination of exemptions and the improvement of tax administration. However, for capacity reasons, these recommendations will need to be sequenced over time. In 2011, the authorities plan notably to introduce excise taxes on alcohol and tobacco to further broaden the tax base, and also a single flat turnover tax to simplify the taxation of the informal sector.

As they pursue their infrastructure investment program, my authorities will give priority to further strengthening public financial management. They have undertaken a reform in this area that would help improve project selection, reinforce project implementation and enhance monitoring.

The Congolese authorities are aware that, in order to accelerate the diversification of the economy, additional investments in infrastructure alone will not suffice. It will be crucial to improve the business climate and attract investors - both foreign and domestic - to help promote activities in areas outside the oil sector. They are addressing this issue through a comprehensive action plan adopted by the government earlier this year. They thank their 
development partners for the assistance provided in this area, notably the EU, as their involvement was crucial in particular in drafting consumer protection and competition laws, drawing up arbitration manuals, and establishing a one-stop shop for business registration.

\section{Debt Sustainability Analysis}

Since reaching the completion point of the HIPC Initiative early last year, Congo's external position has improved markedly. In order to preserve external stability following the substantial debt relief granted under the HIPC Initiative and MDRI, my Congolese authorities are committed to maintaining a prudent debt strategy. As agreements have been signed with the vast majority of creditors, my authorities remain committed to working toward resolving outstanding debt issues with the remaining few creditors.

My authorities welcome the findings of the DSA, that Congo is at a low risk of debt distress and that debt dynamics are robust to the potential adverse shocks that could affect the country. They share the view on the need to preserve fiscal sustainability and pursue economic diversification as a way to shield public finances from oil price volatility.

\section{Conclusion}

In light of my authorities' continued satisfactory progress under the Fund-supported program, I would like to request the Board's support for the completion of the fifth and sixth reviews under the ECF.

Having reached the final review under the current arrangement, I would like, on behalf of my Congolese authorities, to express my deep appreciation to the Board and Management for the support provided over the years and the valuable recommendations made.

In the period ahead, my authorities will remain closely engaged with the Fund. 